\title{
Inter-dye hole transport accelerates recombination in dye sensitized mesoporous films
}

\author{
Davide Moia, ${ }^{1} *$ Anna Szumska, ${ }^{1}$ Valérie Vaissier, ${ }^{1 * *}$ Miquel Planells, ${ }^{2}$ Neil Robertson, ${ }^{2}$ Brian C. \\ O’Regan, ${ }^{3}$ Jenny Nelson, ${ }^{1}$ Piers R. F. Barnes ${ }^{1 *}$ \\ ${ }^{1}$ Blackett Laboratory, Imperial College London, Prince Consort Road, London SW7 2AZ, UK \\ ${ }^{2}$ EaStCHEM School of Chemistry, University of Edinburgh, King's Buildings, David Brewster Road, \\ Edinburgh EH93FJ \\ ${ }^{3}$ Department of Chemistry, Imperial College London, London SW7 2AZ \\ *davide.moia11@imperial.ac.uk; piers.barnes@imperial.ac.uk \\ ** Current address: Department of Chemistry, Massachusetts Institute of Technology, 77 \\ Massachusetts avenue, Cambridge, MA 02139, USA
}

\begin{abstract}
Charge recombination between oxidized dyes attached to mesoporous $\mathrm{TiO}_{2}$ and electrons in the $\mathrm{TiO}_{2}$ was studied in inert electrolytes using transient absorption spectroscopy. Simultaneously, hole transport within the dye monolayers was monitored by transient absorption anisotropy. The rate of recombination decreased when hole transport was inhibited selectively, either by decreasing the dye surface coverage or by changing the electrolyte environment. From Monte Carlo simulations of electron and hole diffusion in a particle, modelled as a cubic structure, we identify the conditions under which hole lifetime depends on the hole diffusion coefficient for the case of normal (disorder free) diffusion. From simulations of transient absorption and transient absorption anisotropy, we find that the rate and the dispersive character of hole transport in the dye monolayer observed spectroscopically can be explained by incomplete coverage and disorder in the monolayer. We show that dispersive transport in the dye monolayer combined with inhomogeneity in the $\mathrm{TiO}_{2}$ surface reactivity can contribute to the observed stretched electron-hole recombination dynamics and electron density dependence of hole lifetimes. Our experimental and computational analysis of lateral processes at interfaces can be applied to investigate and optimize charge transport and recombination in solar energy conversion devices using electrodes functionalized with molecular light absorbers and catalysts.
\end{abstract}

\section{Introduction}

The photo-generation and separation of charges at the interface of molecular systems represents the core energy conversion process of dye sensitized solar cells (DSSCs) and solar fuel systems. ${ }^{1,2}$ The efficiency of these devices is reduced by fast recombination of electrons in the oxide and holes in the photo-oxidized dyes. Therefore, it would be beneficial to design systems with long charge lifetime (i.e. slow recombination). ${ }^{3,4}$ 
Investigation of the recombination process between electrons in titanium dioxide $\left(\mathrm{TiO}_{2}\right)$ and holes on surface anchored dyes is typically performed by characterizing films immersed in inert electrolytes. This guarantees that photo-generated holes are not transferred to the electrolyte and remain in the dye monolayer until they recombine with photo-generated electrons. Transient absorption spectroscopy (TAS) studies showed that electron hole recombination kinetics in dye sensitized $\mathrm{TiO}_{2}$ nanocrystalline films in inert electrolytes depends on the electron density in the $\mathrm{TiO}_{2}$ electrode. ${ }^{15,6}$ However the decay kinetics indicated that the recombination occurred with a wide distribution of time constants (a dispersive process) and could not be described by a simple second order model. These experimental observations were reproduced with simulations using a continuous time random walk (CTRW) model where the recombination process is limited by trapping-detrapping mediated transport of electrons in the $\mathrm{TiO}_{2}$ reaching surface anchored oxidized dyes. ${ }^{5,7}$ The model assumed an exponential density of trap states below the conduction band in $\mathrm{TiO}_{2}$ described by the characteristic energy $E_{c h}$. The results from this model, used to describe the TAS decay of the photo-oxidized dye population, could also be analytically approximated by a stretched exponential function with stretching parameter $\alpha=k_{B} T / E_{c h}$ (where $k_{B} T$ is the thermal energy). ${ }^{6}$ Those studies identified that the half-life of the photogenerated holes, $t_{50 \%}$, varied with the background electron density, $n$, according to $t_{50 \%} \propto n^{-1 / \alpha}$. Subsequent studies examined the influence of the chemical structure of the dye on the kinetics of the electron hole recombination reaction. The results presented evidence for systems showing longer lifetimes due to reaction limitations as opposed to electron transport limitation. ${ }^{8,9,10,11}$ Thus, tuning surface properties $^{12,13}$ and modifying the dye chemical structure ${ }^{9}$ have been emphasized as routes to minimise electron-dye recombination. On the other hand, other reports showed unchanged recombination dynamics upon change in dye chemical structure, suggesting that diffusion limited encounter between electrons and holes represents the rate limiting process in some systems. ${ }^{14,15}$ The studies described above focussed on the transport of the electrons and on dye-surface interaction while assuming holes to be fixed on dye molecules after photo-generation.

However, hole transport between dyes anchored to the surface of mesoporous films has been demonstrated via electrochemical and spectroscopic techniques for sensitized films when the coverage of dyes on the surface exceeds a percolation threshold of about 50\%. ${ }^{16,17}$ This hole transport process has been applied to solar fuel systems, ${ }^{18,19}$ batteries ${ }^{20}$ and electrochromic devices. ${ }^{21}$ In addition, we have recently shown that it plays a critical role in the photo-conversion of solid state DSSCs, enabling efficient regeneration of the device despite incomplete coverage of the dyed mesostructure by the hole transporting material (HTM) ${ }^{22}$ We have also demonstrated that it is possible to create working DSSCs that do not require a separate HTM since hole collection can be accomplished solely by the dye layer. ${ }^{23}$ The possible influence of hole transport within dye monolayers on hole recombination with electrons in $\mathrm{TiO}_{2}$ has been considered by Ogawa et al.. Electron hole recombination for dye sensitized $\mathrm{TiO}_{2}$ nanocrystalline films was measured using TAS for a series of organic dyes. ${ }^{24}$ Decrease in 
recombination kinetics was observed when introducing non-conjugated side groups to the dyes' chemical structure. An increase in lifetime was also observed upon decreasing the dye surface coverage using the coadsorbent chenodeoxycholic acid (DCA). These results were attributed to the expected variation in the rate of hole transport in different dye monolayers. Other studies that consider the possibility for hole transport to play a role in the recombination dynamics have been reviewed in reference ${ }^{4}$.

In this work we measure the electron hole recombination kinetics using TAS and simultaneously measure hole diffusion in the dye monolayer using transient absorption anisotropy spectroscopy for dye sensitized $\mathrm{TiO}_{2}$ nanocrystalline films immersed in inert electrolytes. We compare samples in which we control the rate of hole transport in the monolayer either by changing the dye surface coverage or the solvent surrounding the sample during the measurement. We also use Monte Carlo simulations to investigate the effect of different monolayer properties on the simulated transient anisotropy profile to aid the interpretations of the experimental results.

This study reveals that hole transfer between dyes influences charge recombination at heterogeneous interfaces and impacts the optimization of photo-electrochemical systems such as DSSCs and solar fuel devices. In addition, our joint experimental and computational approach represents a direct measure of charge transport properties of molecular semiconductors.

\section{Results and discussion}

\section{Electron-hole recombination: the effect of dye surface coverage}

Figure 1a and $\mathrm{b}$ show transient measurements of oxidized dye absorption following $450 \mathrm{~nm}$ wavelength laser pulse excitation of $\mathrm{D} 131$ sensitized $\mathrm{TiO}_{2}$ electrodes with different dye surface coverage. The variation in transient optical density $(\Delta \mathrm{OD})$ reflects the population of oxidized dyes remaining, probed at $1000 \mathrm{~nm}$ wavelength, and hence the dynamics of hole recombination with electrons. The choice of wavelengths is discussed in Section 1 of the Supporting Information. Data are plotted for different values of the background electron concentration in $\mathrm{TiO}_{2}, n$, controlled electrochemically (see figure 1e) and measured via charge extraction. The measurements were performed on the sample immersed in an inert electrolyte $(0.1 \mathrm{M}$ tert-butyl ammonium perchlorate in acetonitrile) in a three electrode cell as discussed in the experimental section. The lifetime of the holes decreases substantially with increasing $n$ in both cases of (a) high and (b) low D131 coverage, consistent with previous reports. ${ }^{5}$ Figure 1c compares the half-life of the fully dyed D131 sensitized sample with samples where the D131 loading was decreased either by using a short dyeing time or by using the coadsorbent DCA to limit the uptake of the dye to the surface. ${ }^{25}$ The case of partial dye desorption is also presented in Section 2 of the Supporting Information. 

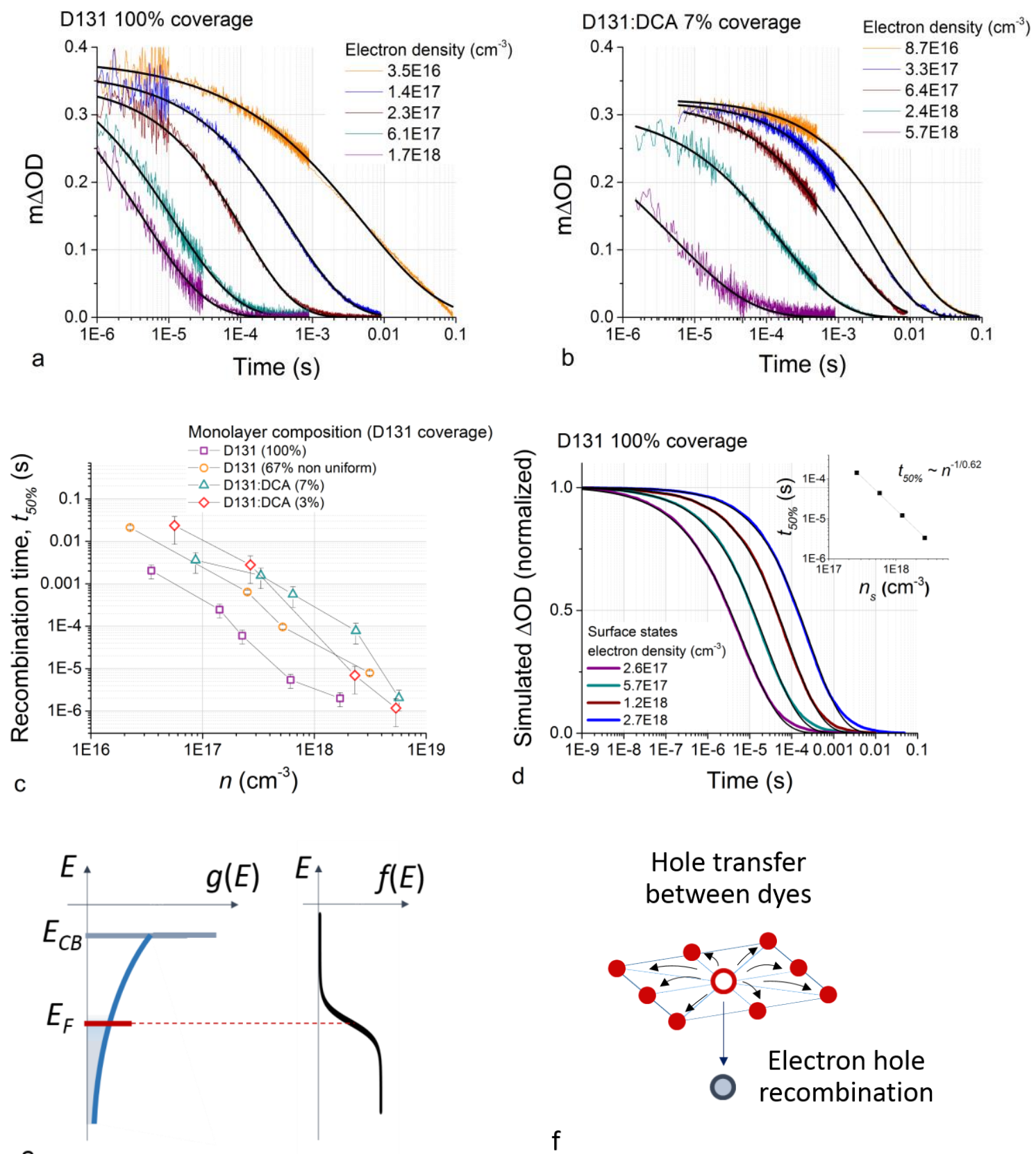

Figure 1. Transient absorption spectroscopy decays of (a) D131 and (b) D131:DCA sensitized $\mathrm{TiO}_{2}$ nanocrystalline films in $0.1 \mathrm{M}$ TBAP in acetonitrile. The measurements refer to different background electron concentration in the $\mathrm{TiO}_{2}, n$, induced by the applied bias and calculated from charge extraction measurement (see experimental section). The samples were pumped with laser pulses at a wavelength of $450 \mathrm{~nm}$ and the oxidized dye population was probed at $1000 \mathrm{~nm}$. (c) Half-life times as a function of electron concentration in the $\mathrm{TiO}_{2}$ for $\mathrm{D} 131$ sensitized $\mathrm{TiO}_{2}$ samples with full dye coverage (purple) and with decreased dye coverage. The low dye coverage was obtained by either short dyeing time, which we expect to yield non uniform dyeing, or D131:DCA co-sensitization. The slope of $\log \left(t_{50}\right)$ vs $\log (n)$ varies 
between $-(0.44)^{-1}$ and $-(0.62)^{-1}$ for the different samples. The coverage fractions refer to D131 and were determined via dye desorption, as described in Section 3 of the Supporting Information. Error bars were calculated based on the fractional variation in $t_{50 \%}$ of TAS decays taken at open circuit before and after all the measurements. (d) Simulated hole recombination in D131 monolayer with electrons situated on the surface of $(20 \mathrm{~nm})^{3} \mathrm{TiO}_{2}$ particles. The black lines correspond to stretched exponentials fitted to the simulations. (e) Schematics of the density of states $g(E)$ in $\mathrm{TiO}_{2}$ films and Fermi-Dirac distribution $\mathrm{f}(E)$ as a function of electron energy. The position of the Fermi level $E_{F}$ determines the background electron concentration $n$ in the film. It is controlled with an applied bias in our experiments and it is an input parameter in our calculations. (f) Model of recombination used for the calculations displayed in figure 1d. Hole transfer between dyes competes with recombination with electrons in the $\mathrm{TiO}_{2}$.

Figure 1c shows that hole lifetimes are consistently shorter for the sample with full coverage than for samples with decreased dye loading, even for concentrations of photo-generated electrons much lower than the total electron density ( $n_{p h}<n / 10$, see experimental section). This shows that, for D131 sensitized $\mathrm{TiO}_{2}$ samples at equal electron densities, high dye loading correlates with faster electron hole recombination. The trend is observed up to electron concentration exceeding $10^{18} \mathrm{~cm}^{-3}$, which is representative for DSSC devices operating at maximum power point under 1 sun illumination. ${ }^{26}$ Also, the recorded recombination lifetimes in this region are in the order of microseconds. This is comparable to the kinetics of regeneration observed in DSSCs using redox couples which enable high open circuit voltage $\left(V_{o c}\right)$ solar cells. ${ }^{27}$ The recombination between holes on dyes and electrons in $\mathrm{TiO}_{2}$ can therefore become important in solar cell devices under operating conditions.

We note that molecular orientations on the $\mathrm{TiO}_{2}$ may be sensitive to the dye surface coverage conditions as well as to the presence of the inert coadsorbent. Although were not able to control this orientation experimentally, we expect that high surface packing in the dye monolayer may lead to an increase in the distance between photo generated holes from the $\mathrm{TiO}_{2}$ surface. This would potentially result in slower interfacial reaction kinetics. ${ }^{9}$ Since the high dye coverage case showed faster recombination than the lower coverage cases it is unlikely that molecular orientation plays a dominant role in the differences in recombination dynamics we observed.

In the next sections we investigate the hole transport properties of D131 sensitized samples and discuss how this process could contribute to recombination dynamics. In addition, we simulated the hole recombination kinetics for different electron densities using a model that includes the hole transport properties of the dye monolayer (see schematics in figure 1e and 1f). The data are displayed in figure $1 \mathrm{~d}$ and will also be discussed in the next sections. 


\section{Hole transport in the dye monolayer: interpreting transient anisotropy measurements}

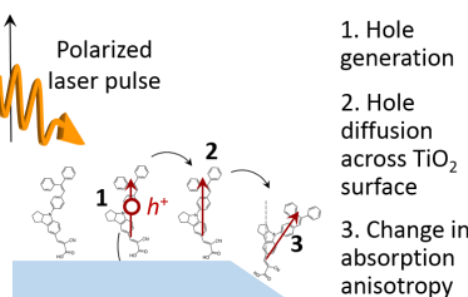

a

Experimental anisotropy $\left(r_{\text {exp }}\right)$
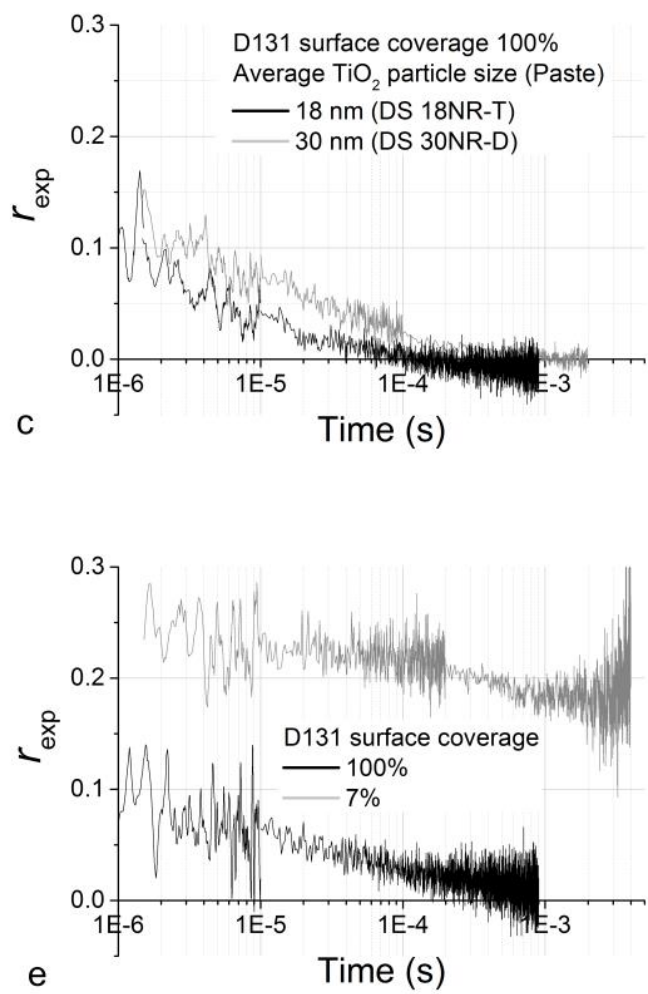

e

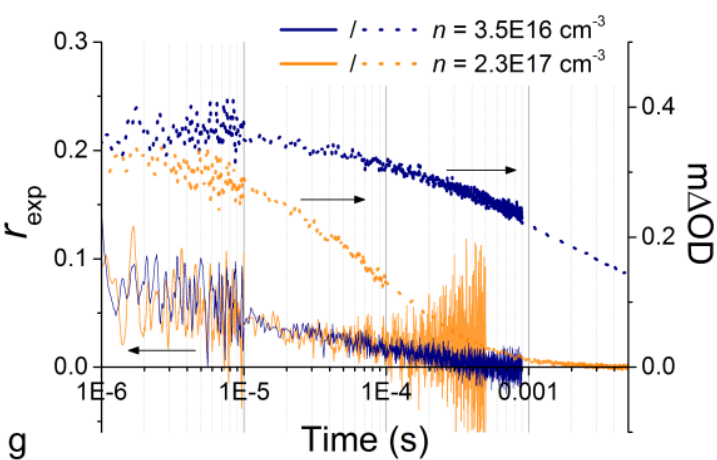

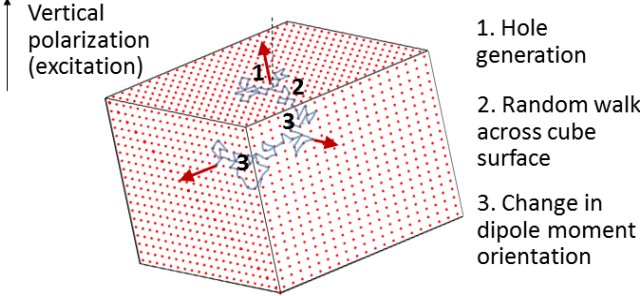

b Simulated anisotropy $\left(r_{\text {sim }}\right)$
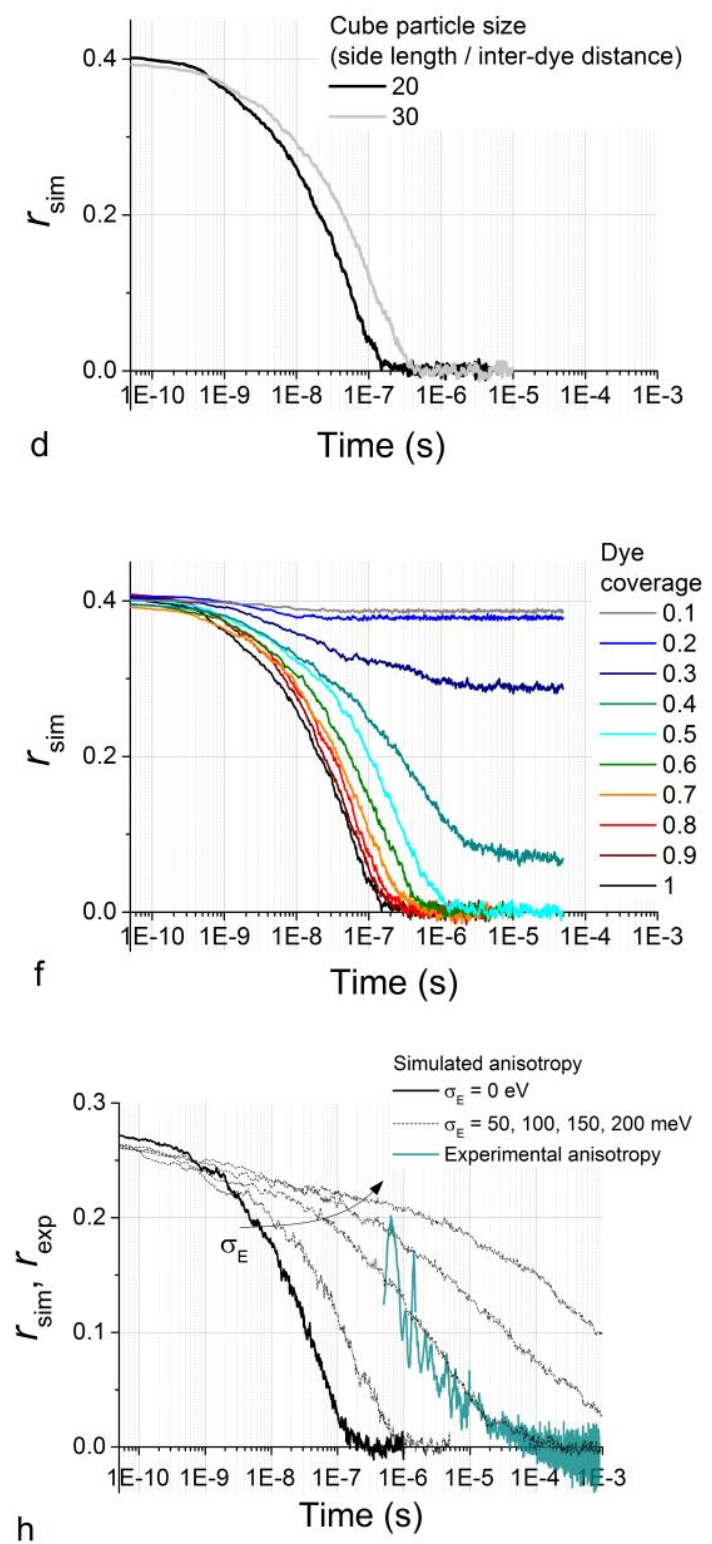

Figure 2. Schematics of (a) experimental and (b) simulated transient absorption anisotropy spectroscopy on D131 sensitized $\mathrm{TiO}_{2}$ nanocrystalline films in $0.1 \mathrm{M}$ TBAP in acetonitrile. (c) Experimental and (d) simulated anisotropy decay for fully dyed $\mathrm{D} 131 \mathrm{TiO}_{2}$ with different 
$\mathrm{TiO}_{2}$ average particle size (diameter). (e) Anisotropy decay measured for films with full (100\%) D131 coverage and with partial (7\%) D131 coverage obtained by co-sensitizing with D131:DCA. The TAS decay for these samples is shown in figure 1a and b. (f) Simulated coverage dependence of transient absorption anisotropy. (g) Anisotropy (left axis) and TAS (right axis) decays measured for a fully dyed $\mathrm{D} 131 \mathrm{TiO}_{2}$ film with different electrochemically induced electron concentrations in the $\mathrm{TiO}_{2}$ film (see legend). (h) Comparison between experimental anisotropy and simulated anisotropy decays for the complete dye coverage case including the effect of energetic disorder in the simulation $\left(\sigma_{\mathrm{E}}\right.$ is the standard deviation of the Gaussian distribution used to assign site energies). The simulated transient absorption anisotropy decays refer to dyes arranged on the surface of a cube where each facet has $20 \times 20$ available sites. Values of electronic coupling $J=18 \mathrm{meV}$ and reorganization energy $\lambda=1.062$ $\mathrm{eV}$ were used in the model. For simulations displayed in (d) and (f) we considered the case of transition dipole moments oriented perpendicular to the cube surface. Simulations shown in (h) considers an angle of $152.6^{\circ}$ between the unoxidized dye's transition dipole moment (oriented perpendicular to the surface) and oxidized dye's transition dipole moment, based on our TD-DFT analysis (see Section 1 and 4 in the Supporting Information).

In figure 2 we show measurements of transient absorption anisotropy under the same experimental conditions as the data shown in figure 1 ( $0.1 \mathrm{M}$ TBAP in acetonitrile). We also compare the experimental observations to simulations of the transient absorption anisotropy profile of holes diffusing in a dye monolayer arranged on the surface of a cubic particle (see experimental section). Transient absorption anisotropy spectroscopy involves monitoring time resolved changes in absorption anisotropy of the sample following pulsed polarized photo-excitation. The measurement provides information on the transition dipole moment orientation of the photo-generated species, at a specific probe wavelength, with respect to the excitation polarization. In the particular case of a dye sensitized $\mathrm{TiO}_{2}$ nanoparticle, pumping the sample with polarized light selectively excites a population of dyes whose transition dipole moments are aligned with the pump polarization at the excitation wavelength. Electron injection in the $\mathrm{TiO}_{2}$ leaves these dyes in the oxidized state which present different optical absorption features to the unoxidized molecule. Probing the changes in absorption polarization (transition dipole moment orientation) of the oxidized dyes at a convenient wavelength enables us to learn about the dynamics of photo-generated holes. In our case, the anisotropy probed at $1000 \mathrm{~nm}$ is expected to be dominated by the oxidized D131 component (see Section 1 of the Supporting Information). Previous studies have discussed the relation between the transient anisotropy profile of the oxidized dye absorption and the diffusion of the hole within the monolayer. ${ }^{18,22}$ They concluded that decay in anisotropy can be interpreted in terms of holes being transferred between dyes with different orientation. Changes in such orientation occur when the hole is transferred to dyes that are anchored to sites of a particle with different surface orientations, 
suggesting that experimental anisotropy decays could be directly interpreted in terms of distance travelled by holes. For example, in the case of hole diffusion on a spherical surface where the dyes' transition dipole moments are oriented perpendicular to the surface, anisotropy is expected to decay mono-exponentially. ${ }^{18}$ On the other hand, neighboring dyes can present different orientation due to different molecular configurations with respect to the surface, implying that evaluating the rate of diffusion from transient anisotropy decays requires detailed knowledge of the system (see full discussion in Section 4 of the Supporting Information). In figure 2c we compare the transient anisotropy decay for mesoporous films made with different $\mathrm{TiO}_{2}$ particle sizes (18 versus $30 \mathrm{~nm}$ diameter) both with full coverage of D131. This comparison shows that hole transport in dye monolayers sensitizing larger size particles results in slower anisotropy decay dynamics. Assuming that this correlation is causal, then this suggests that, for $\mathrm{D} 131$ on $\mathrm{TiO}_{2}$, photogenerated holes must cover distances in the order of the particle size to obtain complete decay of this quantity. The numerical model used for figure $2 \mathrm{~d}$ and $2 \mathrm{f}$ considers dyes adsorbed on a cubic particle where dyes on the same facet show identical transition dipole moments orientation. In this case, changes in anisotropy only occur when the hole diffuses across a facet edge. Based on the above observation about particle size dependence, the model should be at least in part representative of the real system. Figure $2 \mathrm{~d}$ compares the simulated decay in anisotropy for different size of the cubic particle, reproducing qualitatively the difference in the profiles obtained for the experimental results. The factors influencing the profile (amplitude, dispersion and timescale of the decay) of the simulated anisotropy will be considered further below.

Figure 2e shows that, for a fully dyed sample, the transient anisotropy signal decays within few hundreds of microseconds. On the other hand, when the D131 coverage is low, the transient anisotropy signal at the earliest time resolved is about 0.25 and shows incomplete decay up to millisecond timescale. The values of anisotropy recorded are lower than 0.4 which is the theoretical maximum value for isotropic samples. 0.25 is likely to be the highest anisotropy achievable for this dye, due to the relative orientation of the transition dipole moments that are involved in the excitation of the dyes and probing of the oxidized dyes (more details can be found in Section 1 and 4 of the Supporting Information). Slow decay in anisotropy is observed also for the other low coverage samples (see Section 2 of the Supporting Information). These results are in qualitative agreement with figure $2 \mathrm{f}$ where the simulated anisotropy profiles follow different dynamics depending on the surface coverage. In particular for surface coverage $<50 \%$ incomplete decay is observed. This is consistent with confinement of some holes on isolated islands of dyes on particular surface orientations of the particles.

Figure $2 \mathrm{~g}$ shows measurements of transient absorption anisotropy and the respective TAS decay on a D131 sample while applying two different electrochemical potentials to the $\mathrm{TiO}_{2}$ electrode, which result in different background electron concentrations measured by charge extraction. When 
increasing the electron concentration by a factor of 6.5 , the anisotropy decay shows no noticeable difference even though the lifetime of the holes is significantly decreased (by a factor of 33). This implies that electrons in the $\mathrm{TiO}_{2}$ do not significantly influence the rate of hole transport in the dye monolayer. We therefore assume that, for all the traces displayed in figure 1a, hole diffusion kinetics in the dye monolayer are the same.

The stretching shown for simulated transient absorption anisotropy of full coverage samples (figure $2 \mathrm{~d}, \mathrm{f}$ ) is much less pronounced than the one observed experimentally (see figure $2 \mathrm{c}, \mathrm{e}, \mathrm{g}$ ). From figure $2 \mathrm{f}$, we note that dispersion increases slightly when less than $100 \%$ coverage is considered and becomes important when approaching 40-50\% coverage. Interconnection between sites occupied by dyes becomes poor and hinders the diffusion of the charges across the particle's surface. The transition between the conditions of well and poorly interconnected network of dyes can be defined as the percolation threshold. We conclude that local incomplete coverage may contribute to the observed dynamics. In addition, other sources of dispersion in time constant are likely to play a role in determining the stretched profile of the transient absorption anisotropy. In particular, we identify the following main factors: disorder in dye site energies (figure $2 \mathrm{~h}$ ) and in relative spacing or interaction between neighboring dyes; distribution in the relative orientation of the dyes' transition dipole moments; distribution in size and shape of the nanoparticles used experimentally (see full discussion of these factors in Section 4 of the Supporting information). We also show that energetic disorder in the monolayer results in an overestimate of the 'apparent' reorganization energy for intermolecular charge transfer (see figure S7). This observation is expected for transport in disordered systems and represents a potential explanation for the discrepancy between measured and calculated values of reorganization energy in our earlier report. ${ }^{28}$

On the basis of these results and our previous investigations, our current interpretation of the hole hopping process in the (D131) dye monolayer is as follows: hole transport between dyes anchored to the surface of $\mathrm{TiO}_{2}$ particles occurs in samples with dye coverage exceeding the percolation threshold and does not significantly depend on the electron density in the $\mathrm{TiO}_{2}$ scaffold. The stretched anisotropy decays (figure 2) indicate pronounced dispersion in the transport of holes in the dye monolayer across different surface orientations of the nanoparticle. This can be explained by considering local incomplete coverage and/or energetic disorder between different sites. The latter factor increases the apparent activation energy for transport. This can be extracted from temperature dependent electrochemical measurements. Dye fluctuation may be responsible for the relatively high hole diffusion coefficient and electronic coupling that we extract from cyclic voltammetry. ${ }^{29}$ Finally, below the percolation threshold, diffusion of the holes on the surface is hindered due to the limited number of connected paths formed by the dyes. This interpretation enables us to simulate electron hole recombination accounting for hole diffusion properties of the dye monolayer as we discuss in the next sections. 


\section{Electron-hole recombination and hole transport between dyes}

We now consider the mechanism by which lateral hole transport described in the previous section and figure 2 could influence the trend in recombination displayed in figure 1.

Our interpretation of the transient anisotropy measurements shown in this study suggests that the trend of increasing hole lifetime with decreasing dye coverage seen in figure 1c can be explained in terms of slower hole transport, i.e. inhibiting hole transport by reducing the dye surface coverage correlates with decreased rate of electron-hole recombination. This implies that, in addition to electron transport in the $\mathrm{TiO}_{2}$ and interfacial electron-hole reaction, the dynamics of hole transport could be contributing to the observed kinetics of the decay in photogenerated hole population measured with TAS.

The relation between charge transport properties and recombination kinetics in a system depends on the dimensionality of the phases populated by the charges and on the type of 'interface' between such phases where recombination occurs. For example, when electrons and holes share the same phase, the recombination rate constant is expected to depend on the sum of the charge mobilities (Langevin recombination). Conversely, when electrons and holes are transported in two separate phases and need to reach an interface in order for recombination to occur, then the rate of collision of electrons with holes is limited by the slowest charge carrier's mobility. ${ }^{30}$ Recombination between electrons in nanoparticles and holes in the dye monolayer adsorbed to the nanoparticle represents another type of system where one of the charge carriers (holes) is permanently exposed to the recombination interface, while the other (electrons) is not.

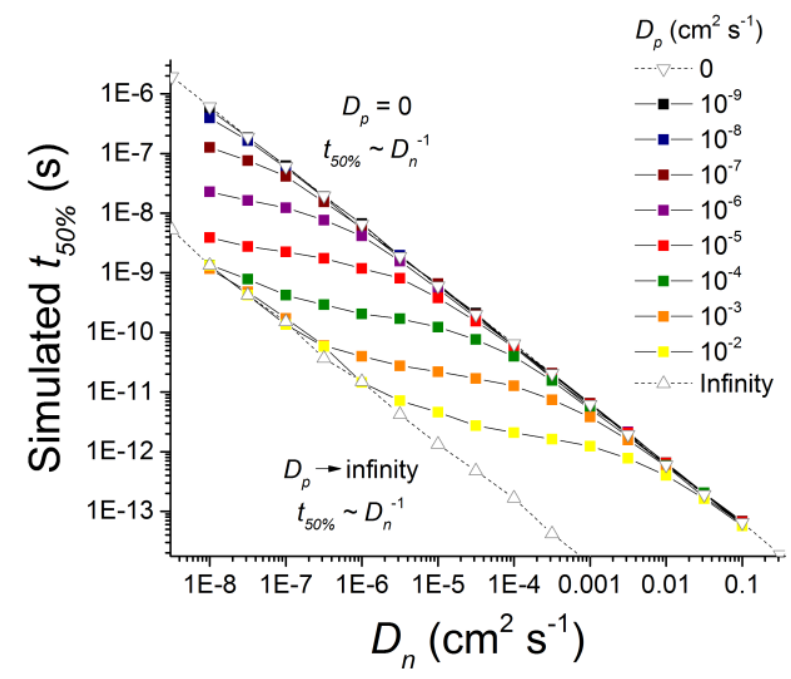

Figure 3. Simulated hole lifetime in a (disorder free) dye sensitized cubic nanoparticle as a function of the electron and the hole diffusion coefficients. 
Figure 3 shows the simulated dependence of hole lifetime on the electron and hole diffusion coefficients for the case of a cubic particle populated with one hole (diffusing on the surface of the cube) and one electron (diffusing within the volume of the cube). For this simulation we considered the case of recombination being limited by collision of the two charges, hence charges recombine when they approach each other within a certain distance (in this case, $\sqrt{3}$ times the lattice parameter).

We can identify three regimes in the recombination kinetics of this system. First, when hole transport in the monolayer is slower than electron transport in the bulk of the particle, recombination is limited by electron diffusion to the interface and to the site where the hole is localized. As a results, $t_{50 \%}$ is proportional to $D_{n}^{-1}$, as it is also expected for the case of immobile holes $\left(D_{p}=0\right)$. When holes move significantly faster in the dye monolayer than electrons within the particle, the recombination time approaches the time that it takes for the electron to diffuse to any site at the interface (since the time taken by the hole to explore the whole particle surface is comparatively negligible). This regime also shows a $t_{50 \%} \sim D_{n}^{-1}$ behavior. In the intermediate regime the hole diffusion coefficient is equal or greater by only few orders of magnitude than the electron diffusion coefficient and the recombination lifetime is dependent on both these quantities.

However, the condition $D_{p}>D_{n}$ is not expected for the case of data presented in figure $1 \mathrm{a}, \mathrm{b}$ and $\mathrm{c}$, at least for the high electron density conditions, therefore this model cannot quantitatively explain our experimental observation. We note that a more complete model is needed to account for the disorder in the $\mathrm{TiO}_{2}$ and in the dye monolayer, which is expected to influence these regimes of electron hole recombination. We address this factor in the next section.

\section{The effect of disorder on recombination}

The effect of energetic disorder and dispersive electron transport in mesoporous $\mathrm{TiO}_{2}$ on the stretched electron-hole recombination kinetics and electron density dependence of holes lifetime has been extensively investigated (see introduction). Below, we discuss the possibility that dispersive transport in the dye monolayer could also contribute to experimental observations of stretched recombination kinetics.

In order to explore this hypothesis, we consider a simplified model for the recombination process where electron transport within the $\mathrm{TiO}_{2}$ particle is not a rate limiting factor. We focus instead on the effect of hole transport and disorder in the dye monolayer on the simulated recombination kinetics. We find that energetic disorder in the dye monolayer combined with inhomogeneity in the $\mathrm{TiO}_{2}$ surface reactivity can introduce significant dispersion in the hole population decay as well as electron density dependence of the hole lifetime which deviates from a first order reaction $\left(t_{50 \%} \sim n^{-1}\right)$. We note that inhomogeneity in surface reactivity of anatase $\mathrm{TiO}_{2}$ nanoparticles is expected, and has been shown previously. ${ }^{31}$ In our model we consider energetic disorder for the $\mathrm{TiO}_{2}$ surface states as a 
source of such inhomogeneity. In figure $1 \mathrm{~d}$ we show the simulated results of electron hole recombination given the model described in the experimental section and in detail in Section 5 of the Supporting Information. These simulations refer to the same hole transport parameters used for the data in figure $2 \mathrm{~h}$ (for $\sigma_{E}=100 \mathrm{meV}$ ) while the energetic disorder in the $\mathrm{TiO}_{2}$ surface is obtained by assigning electronic energy levels drawn from an exponential density of states for each of the cubic particle's surface sites. In the model, the energetic disorder for the surface sites in the $\mathrm{TiO}_{2}$ affects the hole recombination rate to each site, which is calculated to be proportional to the $\mathrm{TiO}_{2}$ electronic site occupancy, given the Fermi level position used in the simulation. The surface site energies and the energetic disorder in the dye monolayer used in our model are uncorrelated. Figure 1d (along with figure S9) shows that the model produces results: with a similar timescale and dispersion to the data displayed in figure 1a for the transient absorption measurement of electron-hole recombination; and with a charge density dependence of the half-life which reproduces the experimental observation. In particular, figure S9 and table S1 of the Supporting Information illustrate that both disorder in the monolayer and in the $\mathrm{TiO}_{2}$ surface reactivity have to be present in order to observe these effects. In the model we have focussed on the case of energetic disorder for both hole transport and the $\mathrm{TiO}_{2}$ surface, however we would potentially expect an analogous trend to result from other sources of disorder.

The only free parameters in the model are the charge transfer rate between electronic states in the $\mathrm{TiO}_{2}$ and oxidized dyes and the position of the conduction band edge. We discuss the sensitivity of the model to these quantities in Section 5 of the Supporting Information. The model reproduces the pronounced deceleration in recombination kinetics when the dye surface coverage is reduced below the percolation threshold observed experimentally.

\section{Hole transport between dyes and electron-hole recombination: the effect of the solvent}
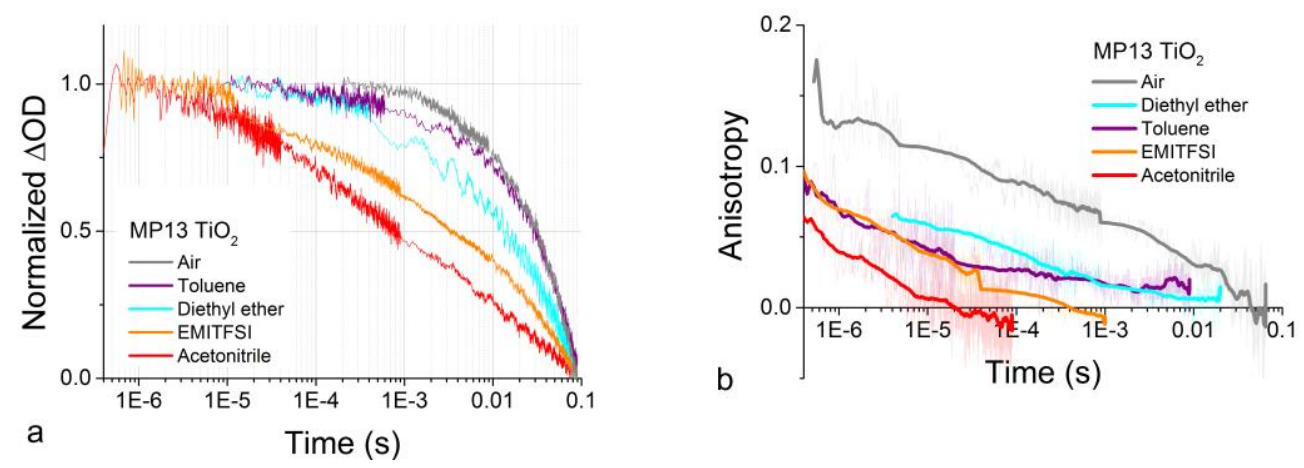

Figure 4. (a) Transient absorption and (b) transient absorption anisotropy spectroscopy on MP13 sensitized $\mathrm{TiO}_{2}$ films on glass immersed in different environments. The films were pumped with pulsed laser excitation at $430 \mathrm{~nm}$ while the oxidized dye signal was probed at $770 \mathrm{~nm}$. 
The solid lines in (b) are obtained by calculating a moving average of the raw data (also displayed in background).

To further investigate the correlation between hole transport in the dye monolayer and electron hole recombination, we measured samples consisting of nominally identical MP13 sensitized $\mathrm{TiO}_{2}$ films (with 100\% dye surface coverage) exposed to different solvent environments. MP13 was chosen as it shows lower degree of desorption when immersed in the solvents investigated and better photostability than D131. Figure 4 shows the TAS and the transient absorption anisotropy decays for holes in MP13 sensitized $\mathrm{TiO}_{2}$ films immersed in 4 different solvents and air. Both the dynamics of electron hole recombination and of hole diffusion undergo a remarkable change when changing the environment. Comparison of the recombination and the transient anisotropy decays between measurements shows that fast anisotropy decay, hence fast hole transport in the dye monolayer, correlates with fast electron hole recombination. One possible explanation for this set of data is therefore that the ability of holes to move on the surface of the particle catalyzes the recombination with electrons in the $\mathrm{TiO}_{2}$. This would be consistent with our interpretation of the results displayed in figure $1 \mathrm{c}$.

We note that the extent to which the transient anisotropy varies when holes hop between neighbouring molecules depends on the relative configuration of the molecules (see Section 4 of the Supporting Information). For this reason, a direct and quantitative comparison of the anisotropy decays in figure $4 \mathrm{~b}$ may not be reliable at this level of description. For the scope of this paper, the comparison of the decay timescales for the different solvent systems is still informative if we simply view the different solvents as a means to empirically change the hole transport rate.

Table 1. Values of viscosity, static dielectric constant $\left(\varepsilon_{r}\right)$ and refractive index taken from reference ${ }^{32,16}$ for EMITFSI and from reference ${ }^{33}$ for other solvents. The value of the Pekar factor $\left(\varepsilon_{e f f^{-1}}\right)$ is calculated as $\varepsilon_{e f f}{ }^{-1}=\varepsilon_{o p t}{ }^{-1}-\varepsilon_{r}^{-1}$, where $\varepsilon_{o p t}$ is the square of the refractive index.

\begin{tabular}{ccccc}
\hline Solvent & $\begin{array}{c}\text { Viscosity }(\mathbf{m P a} \text { ) at } \\
\mathbf{2 5}^{\circ} \mathbf{C}\end{array}$ & $\boldsymbol{\varepsilon r}$ at $\mathbf{2 0}{ }^{\circ} \mathbf{C}$ & $\begin{array}{c}\text { Refractive index } \\
\text { (Temperature) }\end{array}$ & $\varepsilon_{\text {eff }}{ }^{\mathbf{1}}$ \\
\hline Acetonitrile & 0.37 & 36.6 & $1.3442\left(30^{\circ} \mathrm{C}\right)$ & 0.526 \\
Diethyl ether & 0.224 & 4.27 & $1.3526\left(20^{\circ} \mathrm{C}\right)$ & 0.312 \\
Toluene & 0.56 & 2.38 & $1.4961\left(20^{\circ} \mathrm{C}\right)$ & 0.0266 \\
EMITFSI & 88 & $<10$ & $1.4305\left(20^{\circ} \mathrm{C}\right)$ & $0.39<\varepsilon_{\text {eff }}^{-1}<0.49$ \\
Air & 0.019 & 1 & 1 & 0 \\
\hline
\end{tabular}

In table 1, some of the properties of the solvents used are displayed. No clear trend is found between the kinetics of hole transport and the viscosity of the solvent, suggesting that this factor does not play a primary role in the determination of charge diffusion between dyes. We observe that faster dynamics for the transient anisotropy decay correlates with higher Pekar factor $\varepsilon_{e f f}{ }^{-1}$, defined as $\varepsilon_{e f f}{ }^{-1}=\varepsilon_{o p t}{ }^{-1}-\varepsilon_{r}^{-1}$ (where $\varepsilon_{o p t}$ and $\varepsilon_{r}$ are the optical and static dielectric constants of the solvent). This is in contrast with 
what would be expected for inter-dye hole transfer in a solvent environment within the non-adiabatic Marcus theory. When the environment is treated as a continuous medium, the outer sphere reorganization energy for charge transfer is proportional to the Pekar factor, implying that for environments showing high Pekar factors a slower transfer rate would be expected. ${ }^{18,23}$ A similar trend was observed for estimates of the hole diffusion coefficient in dye sensitized oxide films from electrochemical measurements where ions are present in electrolytes. The diffusion coefficient was found to increase using solvents with increasing polarity. ${ }^{16}$ The results were explained in terms of faster charge compensation by the ions in polar solvents upon inter-dye hole transfer where ion pairing is less pronounced than in non-polar solvents. In our experiments, the same trend is found in absence of electrolyte ions, ruling out the role of ionic charge compensation as a limiting step for charge transport for our system. This observation questions the validity of predicting charge transfer rates in these weakly coupled systems on the basis of the expected outer sphere reorganization energies according to non-adiabatic Marcus' theory. Considering the surrounding solvent as a dielectric continuum may represent one key limitation. We also note that the reorganization energy is not the only parameter which is affected by the surrounding medium. Different solvents may influence the configuration of the molecules on the surface, thus influencing the transport rate. In particular, the coupling between dyes is highly sensitive to the packing of the monolayer and eventual change in order. One possibility is that the use of polar solvent results in more favourable configurations of the dyes for charge transport. The change in the electrostatic environment could also vary the energetic disorder in the monolayer.

The different solvents used here are expected to result in different outer sphere reorganization energies of charge transfer between dyes, but also different outer sphere reorganization energies for the electron transfer from $\mathrm{TiO}_{2}$ to oxidized dyes. Assuming that the latter process occurs in the Marcus inverted region, higher reorganization energy would result in faster rates of charge transfer. ${ }^{34,35}$ The results shown in figure 4a could therefore also be interpreted on the basis of increasing Pekar factor resulting in faster recombination reaction upon electron hole encounter. This interpretation assumes that the electron transfer reaction between $\mathrm{TiO}_{2}$ and the dyes is the limiting factor to the rate of recombination, as opposed to the electron transport to the dye- $\mathrm{TiO}_{2}$ interface. On a related note, the solvent surrounding the sensitized film is expected to vary the oxidation potential of the dye. This influences the difference in free energy describing the charge recombination reaction and ultimately its rate. We note that a complete model describing recombination in dye- $\mathrm{TiO}_{2}$ systems as a Marcus' inverted region reaction is still missing. ${ }^{36,37}$ In addition to these factors, the use of different solvents may vary the $\mathrm{TiO}_{2}$ density of states and mobility of electrons in the film. This effect could also contribute to the observed trend.

In a previous report by Bonhôte et al., hole transport through the dye monolayer to the FTO contact in DSSCs was considered as a possible route for faster electron-hole recombination. ${ }^{11}$ In our study 
correlation between hole transport and hole lifetime is observed for samples fabricated on glass substrates or including a $\mathrm{TiO}_{2}$ compact layer covering the FTO contact indicating that hole transfer to electrons in FTO does not contribute to observed trend in recombination kinetics.

\section{Conclusions}

We have shown experimental evidence that hole transport in high coverage dye layers anchored to the $\mathrm{TiO}_{2}$ nanoparticles increases the rate of recombination of photo-generated holes with electrons in the oxide. We demonstrated that reducing the rate of recombination (and the rate of hole transport) can be achieved by either reducing the dye surface coverage or by using solvents showing low Pekar factor. Using Monte Carlo simulations we identified three kinetic regimes of recombination for a cubic particle depending on the relative magnitude of electron and hole diffusion coefficients. The measurements of hole diffusion in the dye monolayer via transient anisotropy spectroscopy suggests that this process is highly dispersive. We simulated the transient anisotropy signal and electron hole recombination using model inputs based on our previous electrochemical and computational investigations of hole hopping between dyes. The observed dispersive character of hole transport can be explained by considering the effect of disorder and incomplete coverage in the monolayer. We also showed that dispersive hole transport, combined with spatial inhomogeneity in interfacial recombination with electrons in the $\mathrm{TiO}_{2}$, contributes to the dispersion in the hole population decay observed with transient absorption measurements and to the electron density dependence of hole lifetime. The significant increase in the lifetime of photo-generated charges that we induced by reducing dye coverage or using solvents with low Pekar factor has important implications for both DSSCs architectures where electron recombination with oxidized dyes limits their $V_{o c}$, and solar fuel devices where surface adsorbates catalyze slow chemical reactions. Together with our previous reports, this study emphasizes the importance of characterizing recombination of photo-generated charges in molecular sensitized mesoporous films in conjunction with the investigation of the charge transport properties of the dye layer. These insights suggest approaches to control intermolecular lateral processes, as opposed to other interfacial properties, as a route to improve device performance. We have also shown that studying lateral transport in dye monolayers is a useful strategy to test the validity of electron transfer theories adopted to model charge transfer events in these systems. Our data show that the solvent dependence of hole transport between dyes cannot be explained on the basis of the expected variation in reorganization energy of charge transfer and the use of non-adiabatic Marcus theory.

\section{Experimental section}

Sample preparation and spectroscopy measurements were carried out using the setup and methods described in references ${ }^{28,38}$ and ${ }^{22}$. These can be found in Section 3 of the Supporting Information. 
The transient anisotropy $r_{\exp }(t)$ was calculated from the measurements of transient optical density for vertical and horizontal polarization of the probe beam $\left(\Delta O D_{\mathrm{V}}(t)\right.$ and $\Delta O D_{\mathrm{H}}(t)$ respectively), following a vertically polarized optical pump pulse, as:

$$
r_{\text {exp }}(t)=\frac{\Delta O D_{V}(t)-\Delta O D_{H}(t)}{\Delta O D_{V}(t)+2 \Delta O D_{H}(t)}
$$

For all the anisotropy measurements shown in this study, $\triangle O D_{V}$ has been calculated as the average of a measurement taken before and after the measurement of $\triangle O D_{H}$, to approximately compensate for any degradation of the film. This was significant for some of the samples as we show in Section 6 of the Supporting Information. TAS and transient anisotropy measurements were performed on D131 (purchased from Mitsubishi paper mills limited) and MP13 (chemical synthesis is illustrated in Section 7 of the Supporting Information) sensitized $\mathrm{TiO}_{2}$. Samples with incomplete D131 coverage were fabricated by either adopting short dyeing time or by using the inert coadsorbent chenodeoxycholic acid (DCA, purchased from Sigma Aldrich). The chemical structure of the dyes and the DCA molecule are shown in figure 5.

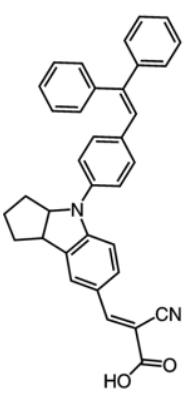

D131

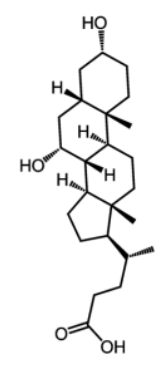

DCA

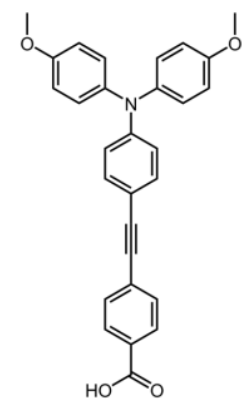

MP13

Figure 5. Chemical structure of the D131 and MP13 dyes and of the inert coadsorbent chenodeoxycholic acid (DCA).

The $\mathrm{TiO}_{2}$ nanoparticle pastes were purchased from Dyesol. The paste $18 \mathrm{NR}-\mathrm{T}$ ( $18 \mathrm{~nm}$ average particle size) was used for all experiments apart from results displayed in figure $2 \mathrm{c}$ where a sample fabricated using the paste $30 \mathrm{NR}-\mathrm{D}$ ( $30 \mathrm{~nm}$ average particle size) is also considered.

The measurements of D131 sensitized samples were carried out at controlled electrical potentials in an electrochemical cell. The cell was sealed and the electrolyte was flushed with argon for 20 minutes before the beginning of the measurements. The flow of argon was left blowing on top of the solution during all measurements to guarantee oxygen free environment. This was done to prevent electrochemically induced or photo-generated electrons reacting with oxygen. The dyed $\mathrm{TiO}_{2}$ sample was used as working electrode in a three electrode configuration, a silver wire and a platinum electrode were used as quasi reference and counter electrodes respectively. Spectroscopy 
measurements were carried out while applying different potential values at the working electrode. For each trace, starting from a potential of $0.2 \mathrm{~V}$ vs silver wire quasi reference electrode, the working electrode was switched to the desired potential (between $0 \mathrm{~V}$ and $-0.5 \mathrm{~V}$ vs silver wire). The TAS measurement was performed under these conditions. The working electrode was then switched back to $0.2 \mathrm{~V}$ at the end of the TAS measurement. The electron density in the film ( $n$ ) was calculated by integrating the measured current density from the last of the three steps. The measured signal is expected to be related to the electron density in the $\mathrm{TiO}_{2}$ during the TAS measurement. A non-zero current was recorded even after long time, suggesting a faradaic component due to electrons exchanged with the electrolyte. The reported values of charge density refer to the integrated current over a constant period of 60 seconds. The current density value at 60 seconds was subtracted to compensate for the baseline faradaic current (see Section 2 of the supporting information). The charge density, $n$, was not corrected for film porosity. The potential of the silver wire was measured before and after each measurement. Fluctuations of its potential with average of $60 \mathrm{mV}$ and ranging between $20 \mathrm{mV}$ and $250 \mathrm{mV}$ were recorded before and after an experiment. By analysing the recombination dynamics as a function of electron density we avoid the possible issue of potential drift for different measurements and between samples. When relating the spectroscopic data to the charge density measurement, we assume that the background concentration of electrons is homogeneous within the $\mathrm{TiO}_{2}$ film, while we expect the profile of photo-generated electrons $n_{p h}$ to vary through the depth of the film. The peak concentration of photo-excited dyes in $\mathrm{D} 131$ sensitized $\mathrm{TiO}_{2}$ films by each laser pulse at the fluence used in the experiment is about $810^{16} \mathrm{~cm}^{-3}$ for the $100 \%$ coverage sample and 2.4 $10^{15} \mathrm{~cm}^{-3}$ for the lowest dye coverage sample. Considering porosity of the film of 0.6 and approximating the $\mathrm{TiO}_{2}$ particles as spheres of $10 \mathrm{~nm}$ radius, these values correspond to about 0.8 and 0.025 photo-excited dyes per particle.

The same spectroscopic characterization measurements (TAS and anisotropy) were made on MP13 sensitized $\mathrm{TiO}_{2}$ films fabricated on glass without controlling the electrochemical potential of the samples.

Stretched exponential functions (equation 2) were fitted to the decays.

$$
\Delta O D_{f i t}(t)=A \exp \left[-\left(\frac{t}{\tau}\right)^{\alpha}\right]
$$

In Eq. 2, $\alpha$ is the stretching parameter and $\tau$ the time constant. Half-life times were calculated from the fits using equation 3.

$$
t_{50 \%}=\tau(\ln 2)^{1 / \alpha} .
$$


For most MP13 samples a background concentration of holes was present during the TAS measurements. This implies that half-life times for the incomplete decays would be longer than the ones that would be extracted from the data presented. Since the accumulation of background charges speeds up otherwise very slow recombination dynamics, this does not vary the relative trend of the kinetics observed between different measurements.

Monte Carlo simulations: simulations of transient absorption anisotropy were carried out considering a square lattice of sites (dye monolayer) on the surface of a cube $\left(\mathrm{TiO}_{2}\right.$ nanocrystal). The data presented in the results section correspond to simulations of hole transfer between D131 dyes on the surface of a cubic $\mathrm{TiO}_{2}$ particle of $20^{3} \mathrm{~nm}^{3}$ in size. Inter-dye distance was approximated to $1 \mathrm{~nm}$. In the model, one hole at a time is considered per simulated experiment. The hole can be exchanged only between sites occupied by dyes and between up to 8 nearest neighbours. The kinetics of hole transfer between available sites are calculated using adaptive waiting times, which is expressed as a function of the non-adiabatic Marcus' charge transfer rate (see Section 3 in the Supporting Information). The reorganization energy of charge transfer was calculated as reported in a previous study, and the case of $0.1 \mathrm{M}$ ions in acetonitrile was considered to match the experimental conditions. ${ }^{39}$ In the disorder free case, the electronic coupling was taken from the experimental estimate reported in reference ${ }^{28}$ (see Section 3 of the Supporting Information for more details). To introduce configurational disorder in the dye monolayer, a Gaussian distribution of calculated electronic couplings between sites was used (the distribution was for $\log J$ ). Similarly, a Gaussian distribution of calculated site energy differences was implemented to simulate energetic disorder in the monolayer.

In order to simulate the transient absorption anisotropy profile expected for photogenerated holes diffusing in the dye monolayer, assumptions on the orientation of the transition dipole moments of the dye have to be taken. Calculations presented in figure $2 \mathrm{~d}, 2 \mathrm{f}$ consider the simplified case where the neutral state and oxidized state transition dipole moments are parallel. Under these conditions, our simulated profiles show initial values of 0.4 , which is the maximum value expected for anisotropy measurement in isotropic systems. The transient anisotropy profile describes the change in orientation of the transition dipole moment of the oxidized dyes population in time. Therefore, the distribution of transition dipole moment orientations for the oxidized dyes on the surface of the particle influences the dynamics of the transient anisotropy decay. By considering all dipoles to be perpendicular to the surface of the particle, changes in anisotropy for each random walk experiment are recorded only upon hole exchange between different facets. Figure $2 \mathrm{~h}$ refer to calculations where the relative orientation of the unoxidized and oxidized D131 dyes are take into account (see TD-DFT calcualtions in Section 1 of the Supporting Information). For this case, the unoxidized transition dipole moment is still set to be perpendicular to the cube surface, while the oxidized D131 dipoles are oriented at $152.6^{\circ}$ from the normal. A rotation about the normal by a random angle is also applied to these dipoles for all dyes at the beginning of all random walk experiments. We discuss these and other aspects of 
accounting for transition dipole moment and dye backbone orientation in in Section 4 of the Supporting Information.

The facet where the hole was placed at $t=0$ for each experiment was picked on the basis of a probability proportional to $\cos ^{2} \vartheta$, where $\vartheta$ is the angle between the polarization axis of the excitation and the normal vector to the facet's surface. When running the random walk experiment, the square projection of the dipole moment of the oxidized dye in the direction parallel and in one of the directions perpendicular to the polarization axis were recorded. These quantities were averaged over time for all experiments and finally used to calculate the simulated anisotropy decay using eq. 1 . The random walk was ended after a certain time. This was the same for all experiments in a simulation. Each experiment takes into account a different orientation of the cubic particle with reference to the polarization axis of the excitation which was instead kept constant. Each simulated anisotropy profile presented here represents the average of $10^{4}$ experiments.

Simulation of electron hole recombination dynamics shown in figure 1d and in Section 5 of the Supporting Information were carried out using a model that considers one surface electronic state for the $\mathrm{TiO}_{2}$ associated to each dye site. The energy of each state is drawn from an exponential distribution based on experimental observation (see charge extraction measurements in Section 2 of the supporting information). All states share the same quasi Fermi level, i.e. we consider electron transport in the $\mathrm{TiO}_{2}$ to be significantly faster than hole transport in the dye monolayer and electronhole recombination, such that all electronic states are in quasi equilibrium. The rate of electron transfer (recombination) from a $\mathrm{TiO}_{2}$ surface electronic state to a hole located on the site associated to that specific state was evaluated as the product of a fixed rate and the Fermi-Dirac probability of occupancy of the electronic state. Such rate was used in the calculation of the waiting time for hole hopping as an additional 'competing' charge transfer route during hole diffusion. This effectively introduces different recombination rates for different position of the holes on the surface of the particle. The electronic energies of the electron surface states and the energies of the dye sites used for hole hopping between neighbouring dyes were recalculated at the beginning of each experiment.

Simulations of electron-hole recombination shown in figure 3 were carried out considering a cubic particle as described above where electrons can diffuse in the bulk of the particle and holes can diffuse on the surface of the particle. Given the input values of the electron and hole diffusion coefficients $\left(D_{n}, D_{p}\right)$, constant charge transfer rates for electrons and holes were calculated using the relations $k_{n}=6 D_{n} / a^{2}$ and $k_{p}=4 D_{p} / a^{2}$, where $a$ is the lattice spacing. The same value of lattice parameter $a$ was used for the bulk (electron diffusion) and the surface of the particle (hole diffusion). Charges could be exchanged between 6 (for electrons) and 4 (for holes) nearest neighbours. The electron and the hole's positions were initially defined randomly within their respective spaces. Recombination times between charges was recorded once the electron and the hole were at a distance 
equal or lower than $3^{1 / 2} a$. We ran $10^{3}$ experiments for each condition and we extracted the lifetime $\left(t_{50 \%}\right)$ from the resulting population decay.

\section{Associated content}

Supporting Information:

TD-DFT calculations and spectroelectrochemical measurement on D131 dye; bias dependent TAS measurements on $\mathrm{D} 131$ sensitized $\mathrm{TiO}_{2}$ films; additional details on experimental and computational method; analysis of dispersion in transient absorption anisotropy decays; simulation of electron hole recombination; dye degradation; MP13 synthesis and properties.

\section{Acknowledgements}

We thank Xiaoe Li, Shogo Mori and Shane Ardo for useful discussions. DM and PB are grateful for EPSRC fellowship EP/J002305/1. NR thanks EPRSC for grant number EP/H040218/1.

\section{References}

(1) O’Regan, B.; Moser, J.; Anderson, M.; Graetzel, M. J. Phys. Chem. 1990, 94 (24), 8720.

(2) Tachibana, Y.; Vayssieres, L.; Durrant, J. R. Nat. Photonics 2012, 6 (8), 511.

(3) Hagfeldt, A.; Boschloo, G.; Sun, L.; Kloo, L.; Pettersson, H. Chem. Rev. 2010, 110 (11), 6595.

(4) Ardo, S.; Meyer, G. J. Chem. Soc. Rev. 2009, 38 (1), 115.

(5) Haque, S. A.; Tachibana, Y.; Klug, D. R.; Durrant, J. R. J. Phys. Chem. B 1998, 102 (10), 1745.

(6) Nelson, J.; Haque, S.; Klug, D.; Durrant, J. Phys. Rev. B 2001, 63 (20), 1.

(7) Nelson, J. Phys. Rev. B 1999, 59 (23), 374.

(8) Clifford, J. N.; Yahioglu, G.; Milgrom, L. R.; Durrant, J. R. Chem. Commun. 2002, 1260.

(9) Clifford, J. N.; Palomares, E.; Nazeeruddin, M. K.; Grätzel, M.; Nelson, J.; Li, X.; Long, N. J.; Durrant, J. R. J. Am. Chem. Soc. 2004, 126 (16), 5225.

(10) Johansson, P. G.; Kopecky, A.; Galoppini, E.; Meyer, G. J. J. Am. Chem. Soc. 2013, 135 (22), 8331.

(11) Bonhôte, P.; Moser, J. E.; Humphry-Baker, R.; Vlachopoulos, N.; Zakeeruddin, S. M.; Walder, L.; Grätzel, M. J. Am. Chem. Soc. 1999, 121 (6), 1324.

(12) Palomares, E.; Clifford, J. N.; Haque, S. a; Lutz, T.; Durrant, J. R. J. Am. Chem. Soc. 2003, 125 (2), 475.

(13) Wenger, B.; Bauer, C.; Nazeeruddin, M. K.; Comte, P.; Zakeeruddin, S. M.; Gratzel, M.; Moser, J.-E. Proc. SPIE 2006, 6325, 1.

(14) Hasselmann, G. M.; Meyer, G. J. J. Phys. Chem. B 1999, 103 (36), 7675.

(15) Wang, D.; Mendelsohn, R.; Galoppini, E.; Hoertz, P. G.; Carlisle, R. a; Meyer, G. J. J. Phys. Chem. B 2004, 108 (42), 16642.

(16) Bonhôte, P.; Gogniat, E.; Tingry, S.; Barbe, C.; Vlachopoulos, N.; Lenzmann, F.; Comte, P.; Grätzel, M. J. Phys. Chem. B 1998, 5647 (97), 1498. 
(17) Hu, K.; Meyer, G. J. Langmuir 2015, 31 (41), 11164.

(18) Ardo, S.; Meyer, G. J. J. Am. Chem. Soc. 2011, 133 (39), 15384.

(19) Brennan, B. J.; Durrell, A. C.; Koepf, M.; Crabtree, R. H.; Brudvig, G. W. Phys. Chem. Chem. Phys. 2015, 17, 12728.

(20) Wang, Q.; Evans, N.; Zakeeruddin, S. M.; Exnar, I.; Grätzel, M. J. Am. Chem. Soc. 2007, 129 (11), 3163.

(21) Wang, Q.; Zakeeruddin, S. M.; Cremer, J.; Bäuerle, P.; Humphry-Baker, R.; Grätzel, M. J. Am. Chem. Soc. 2005, 127 (15), 5706.

(22) Moia, D.; Cappel, U. B.; Leijtens, T.; Li, X.; Telford, A. M.; Snaith, H. J.; O’Regan, B. C.; Nelson, J.; Barnes, P. R. F. J. Phys. Chem. C 2015, 33 (119), 18975.

(23) Moia, D.; Leijtens, T.; Noel, N.; Snaith, H. J.; Nelson, J.; Barnes, P. R. F. Adv. Mater. 2015, 27 (39), 5889.

(24) Ogawa, J.; Koumura, N.; Hara, K.; Mori, S. Jpn. J. Appl. Phys. 2014, 53, 1.

(25) O’Regan, B.; Li, X.; Ghaddar, T. Energy Environ. Sci. 2012, 5 (5), 7203.

(26) Barnes, P. R. F.; Miettunen, K.; Li, X.; Anderson, A. Y.; Bessho, T.; Gratzel, M.; O’Regan, B. C. Adv. Mater. 2013, 25 (13), 1881.

(27) Yang, W.; Vlachopoulos, N.; Hao, Y.; Hagfeldt, A.; Boschloo, G. Phys. Chem. Chem. Phys. 2015, 17, 15868.

(28) Moia, D.; Vaissier, V.; López-Duarte, I.; Torres, T.; Nazeeruddin, M. K.; O’Regan, B. C.; Nelson, J.; Barnes, P. R. F. Chem. Sci. 2014, 5 (1), 281.

(29) Vaissier, V.; Mosconi, E.; Moia, D.; Pastore, M.; Frost, J. M.; De Angelis, F.; Barnes, P. R. F.; Nelson, J. Chem. Mater. 2014, 26 (16), 4731.

(30) Koster, L. J. a; Mihailetchi, V. D.; Blom, P. W. M. Appl. Phys. Lett. 2006, 88 (5), 1.

(31) Gottesman, R.; Tirosh, S.; Barad, H.; Zaban, A. J. Phys. Chem. Lett. 2013, 17 (4), 2822.

(32) Bonhôte, P.; Dias, A.-P.; Armand, M.; Papageorgiou, N.; Kalyanasundaram, K.; Grätzel, M. Inorg. Chem. 1996, 35 (5), 1168.

(33) Lide, D. R. CRC Handbook of Chemistry and Physics, Internet V.; CRC Press, Boca Raton, FL, 2005.

(34) Gould, I. R.; Ege, D.; Moser, J. E.; Farid, S. J. Am. Chem. Soc. 1990, 11 (112), 4290.

(35) Dang, X.; Hupp, J. T. J. Am. Chem. Soc. 1999, 36 (121), 8399.

(36) Moser, J. E.; Grätzel, M. Chem. Phys. 1993, 176 (2-3), 493.

(37) Yan, S. G.; Prieskorn, J. S.; Kim, Y.; Hupp, J. T. J. Phys. Chem. B 2000, 46 (104), 10871.

(38) Anderson, A. Y.; Barnes, P. R. F.; Durrant, J. R.; O'Regan, B. C. J. Phys. Chem. C 2011, 115 (5), 2439.

(39) Vaissier, V.; Barnes, P.; Kirkpatrick, J.; Nelson, J. Phys. Chem. Chem. Phys. 2013, 15 (13), 4804. 
Table of Contents Graphic
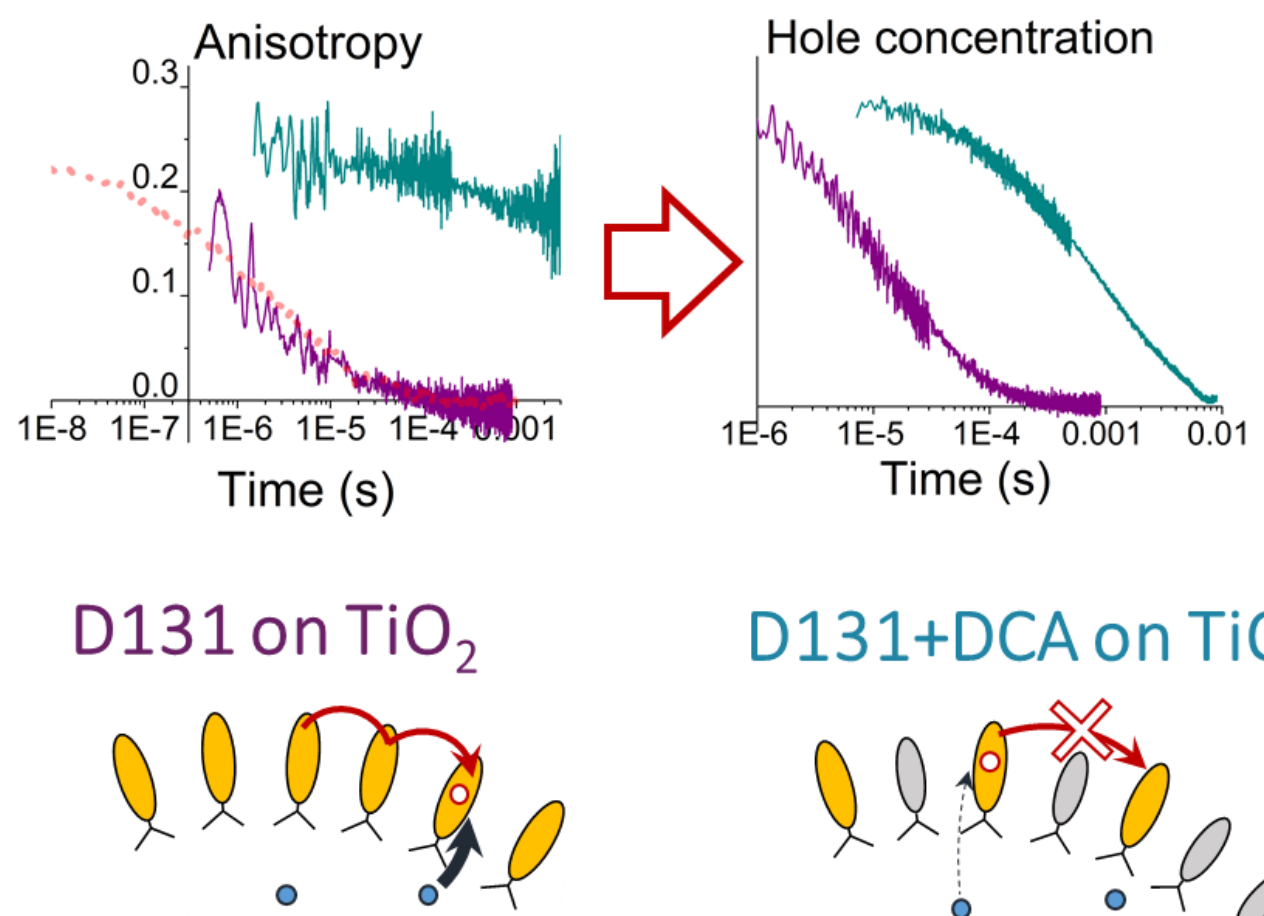

D131+DCA on $\mathrm{TiO}_{2}$

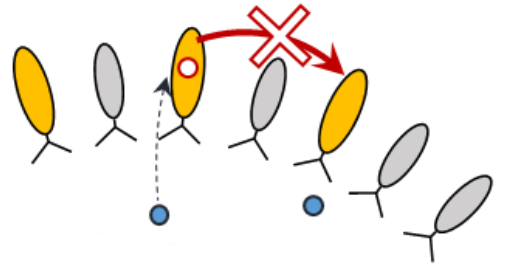


Supporting information

\section{Inter-dye hole transport accelerates recombination in dye sensitized mesoporous films}

Davide Moia, ${ }^{1 *}$ Anna Szumska, ${ }^{1}$ Valérie Vaissier, ${ }^{1 * *}$ Miquel Planells, ${ }^{2}$ Neil Robertson, ${ }^{2}$ Brian C. $\mathrm{O}^{\prime}$ Regan, ${ }^{3}$ Jenny Nelson, ${ }^{1}$ Piers R. F. Barnes ${ }^{1 *}$

${ }^{1}$ Blackett Laboratory, Imperial College London, Prince Consort Road, London SW7 2AZ, UK

2 EaStCHEM School of Chemistry, University of Edinburgh, King's Buildings, David Brewster Road, Edinburgh EH93FJ

${ }^{3}$ Department of Chemistry, Imperial College London, London SW7 2AZ

*davide.moia11@imperial.ac.uk; piers.barnes@imperial.ac.uk

** Current address: Department of Chemistry, Massachusetts Institute of Technology, 77 Massachusetts avenue, Cambridge, MA 02139, USA 


\section{Spectroscopy measurements and TD-DFT calculations on D131}
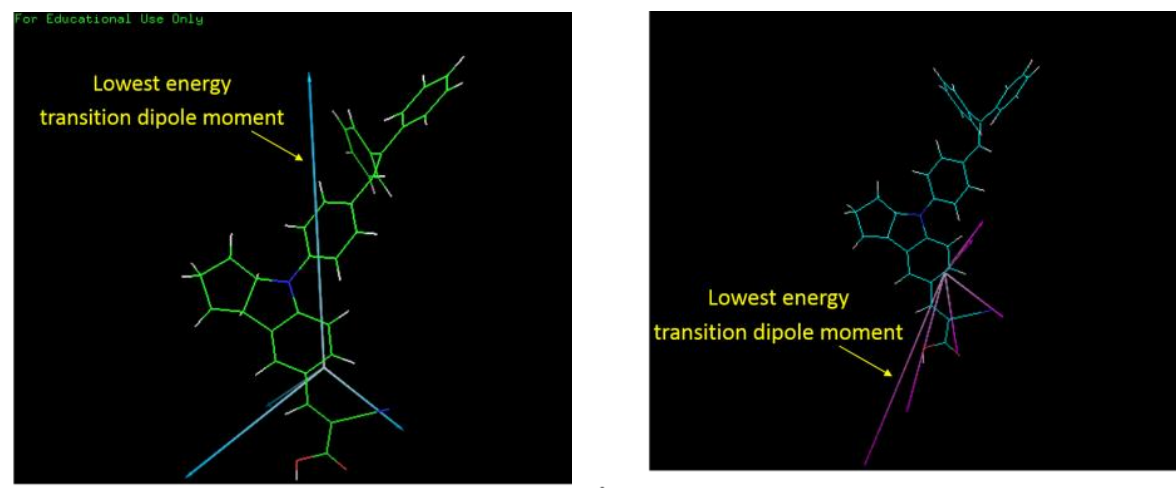

a

b
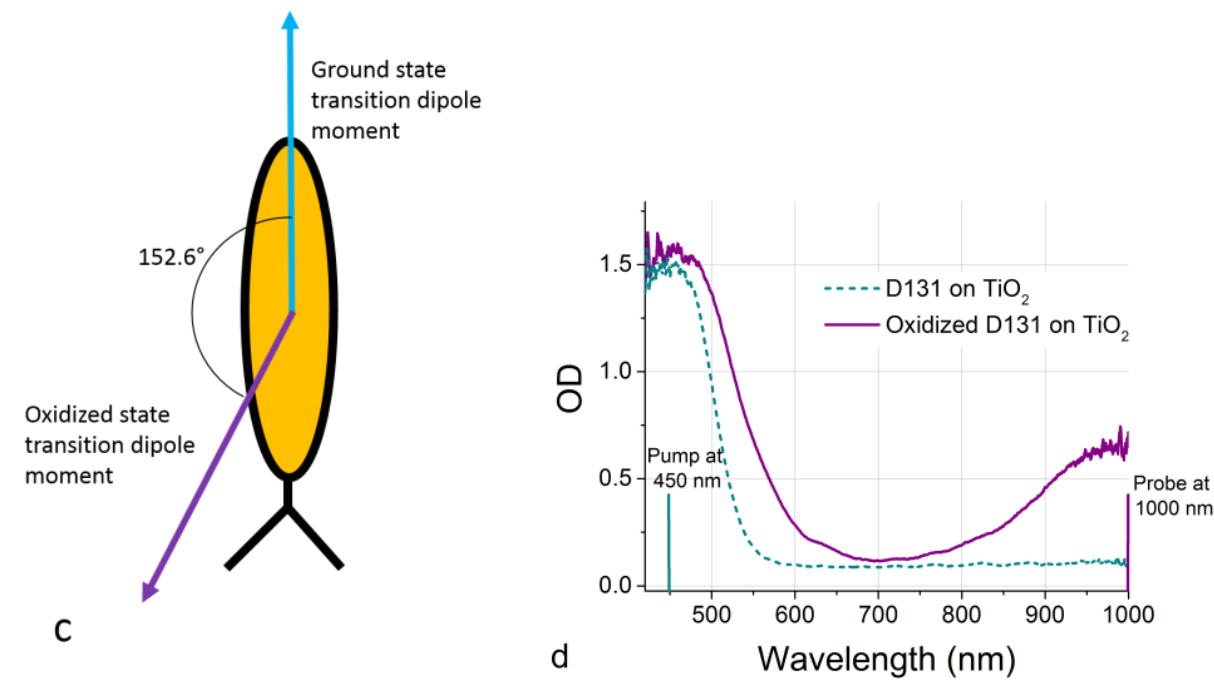

Figure S1. TD-DFT calculations of the transition dipole moments relative to (a) unoxidized D131 and (b) oxidized D131 in acetonitrile. The lowest energy dipoles indicated in the figure are the transitions corresponding to the wavelength of (a) the optical pumping and (b) the optical probe in our experiments. (c) Schematics of the simplified dye configuration and relative position of transition dipole moments unoxidized (light blue) oxidized dye used in our simulations. (d) Absorbance of a D131 sensitized mesoporous $\mathrm{TiO}_{2}$ film deposited on FTO glass immersed in 0.1M TBAP in acetonitrile in the (dashed blue) unoxidized and (purple) oxidized state. Oxidation of the dye was obtained by applying $1.1 \mathrm{~V}$ vs $\mathrm{Ag} / \mathrm{AgCl}$ for 60 seconds to the sample, which was used as working electrode of a three electrode spectroelectrochemical cell. ${ }^{1}$

Figure S1 shows the transition dipole moments of D131 in acetonitrile in its (a) unoxidized and (b) oxidized state calculated using TD-DFT. The unoxidized and oxidized dye molecules were optimized at the DFT level (CAM-B3LYP/TZVP-6D in combination with PCM). TD-DFT calculations were then performed on the optimized geometries (CAM-B3LYP/TZVP-6D with PCM). All quantum chemical calculations were done with Gaussian09. The lowest energy transition dipole moment displayed in figure S1a gives rise to the main absorption window of the dye in the visible (see spectrum of D131 on $\mathrm{TiO}_{2}$ in figure $\mathrm{S} 1 \mathrm{~d}$ ). By pumping our samples at $450 \mathrm{~nm}$ we are expecting to interact predominantly with this transition. The lowest energy dipole displayed in figure S1b corresponds to 
the absorption window of the dye in the oxidized state that we record in the near infrared (see spectrum of oxidized $\mathrm{D}_{131}$ on $\mathrm{TiO}_{2}$ in figure $\mathrm{S} 1 \mathrm{~d}$ ). By probing at $1000 \mathrm{~nm}$ we expect to interact exclusively with this dipole. We note that probing at wavelengths in the visible (for example around $550 \mathrm{~nm}$ ) would make the analysis of anisotropy measurements much more complex since a number of transition dipole moments with different orientations appear to lie in a narrow range of wavelengths. We also note that electrons in the $\mathrm{TiO}_{2}$ also absorb at $1000 \mathrm{~nm}$. However their extinction coefficient (between 1000 and $1500 \mathrm{M}^{-1} \mathrm{~cm}^{-1}$ ) is substantially lower than the extinction coefficient of oxidized D131 (in the order of $20000 \mathrm{M}^{-1} \mathrm{~cm}^{-1}$ ) at this wavelength. ${ }^{1,2}$ The relative orientation of the D131 dye's transition dipole moments corresponding to the pump and probe wavelengths in our experiment is not collinear (angle between the two vectors is $152.6^{\circ}$ ). This implies that the transient absorption anisotropy recorded under these conditions is expected to be less than 0.4 , even before holes are transferred between dyes. ${ }^{3}$ From simulations using the simplified model displayed in figure S1c for dyes anchored to a cubic particle, we obtain a maximum value of anisotropy of about 0.27 , which closely matches experimental observation (see figure $2 \mathrm{~h}$ in main text and Section 4 of this document). We note that in the real system conformational changes may occur upon photoinduced oxidation of the dye on the surface of $\mathrm{TiO}_{2}$, introducing further variation to the angle between the two dipoles. 


\section{Bias dependent TAS measurements}

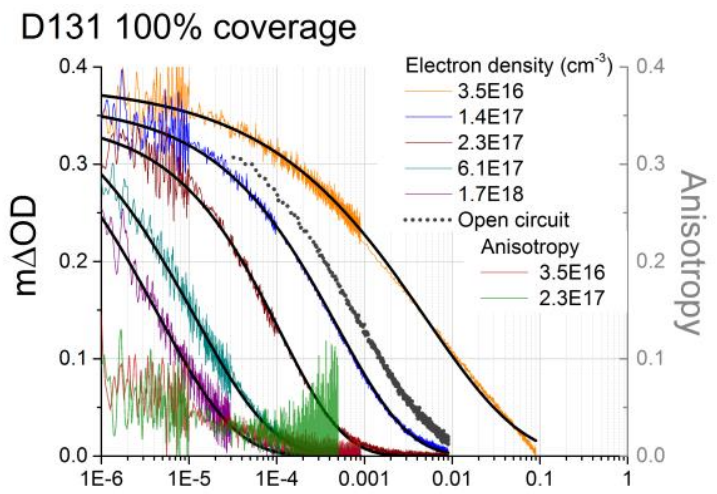

a

Time (s)

D131:DCA 7\% coverage Electron density $\left(\mathrm{cm}^{-3}\right)$

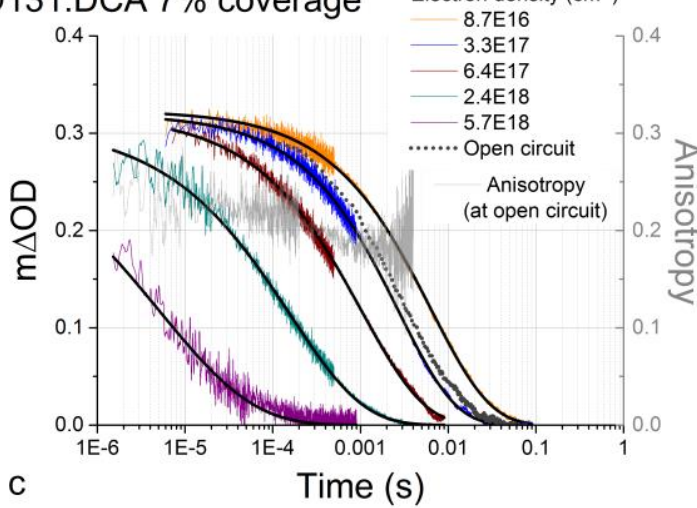

D131 9\% coverage
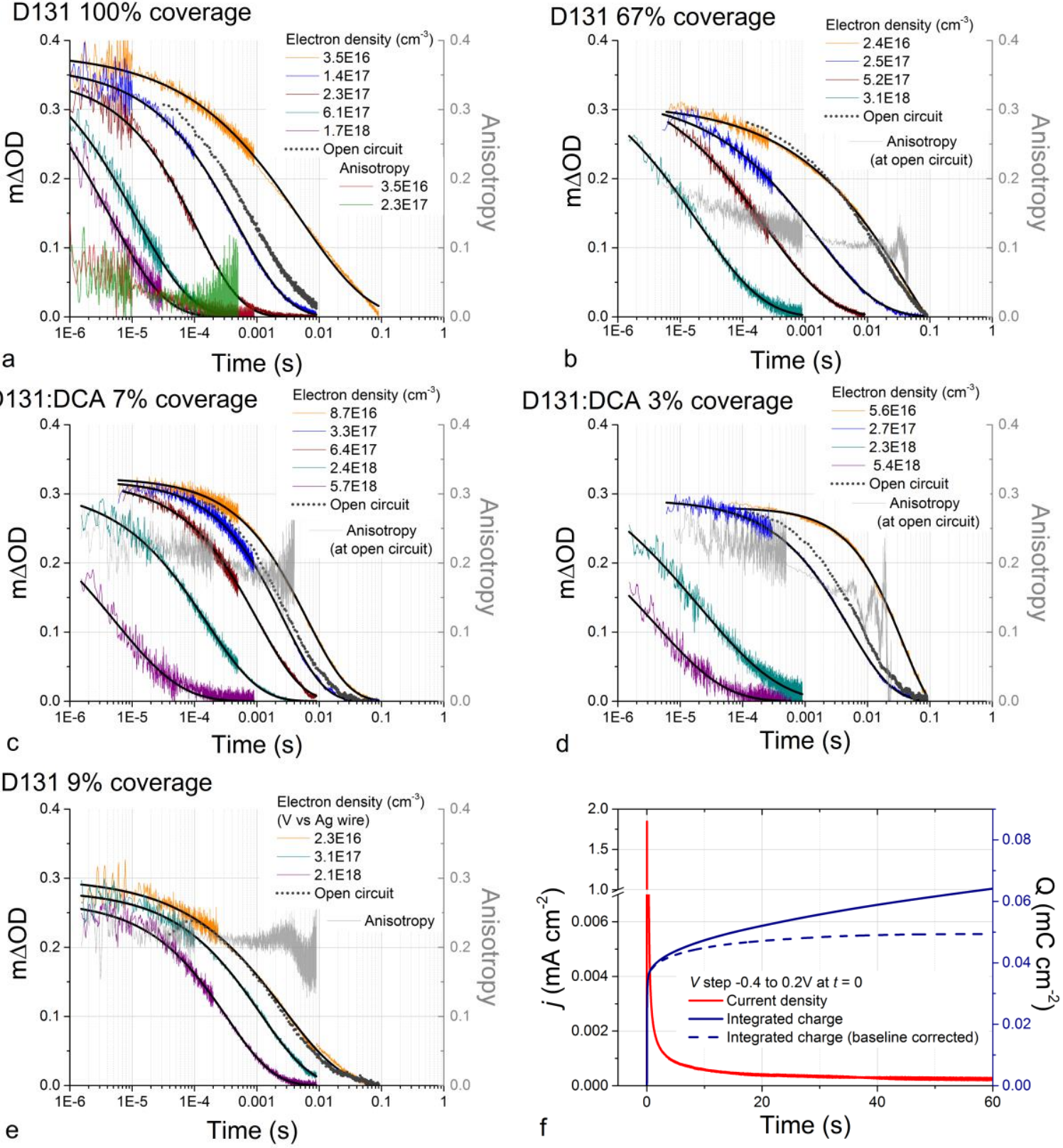

Figure S2. TAS and transient absorption anisotropy measurements of low coverage D131 sensitized $\mathrm{TiO}_{2}$ films measured in 0.1TBAP:acetonitrile electrolyte under controlled electrochemical bias or at open circuit. (a) Full coverage D131 sensitized $\mathrm{TiO}_{2}$ film. The low dye loading was achieved by (b) short dyeing (non-uniform coverage), (c) and (d) cosensitization with the inert molecule DCA and (e) partial dye desorption. (f) Charge extraction measurement performed to evaluate the electron density in $\mathrm{TiO}_{2}$ during the spectroscopy measurements.

Figure S2 shows TAS and transient anisotropy measurements on samples with different dye loading and under different electron density conditions. Measurements at open circuit are also displayed, the recombination timescale seen in these suggest that the electron concentration in the device at open circuit is greater than that injected by the less negative applied bias values where electrons will be removed from the films as photocurrent. We note that the dependence of $t_{50 \%}$ on the electron density for the desorbed sample is much weaker than for all the other cases. This could be due to 
very different surface properties of this film due to exposure to TMAOH during the desorption step. In figure S2f, the evaluation of the charge density in the film is presented: the electrochemical potential is switched to more positive voltage $(0.2 \mathrm{~V}$ vs Ag wire) to enable extraction of electrons from the $\mathrm{TiO}_{2}$ and the current density is integrated over 1 minute. The resulting charge is corrected by a baseline current which is still observed after such time, assuming this is due to a leakage current flowing from the electrode to the electrolyte. The integrated charge and corrected charge are also shown.

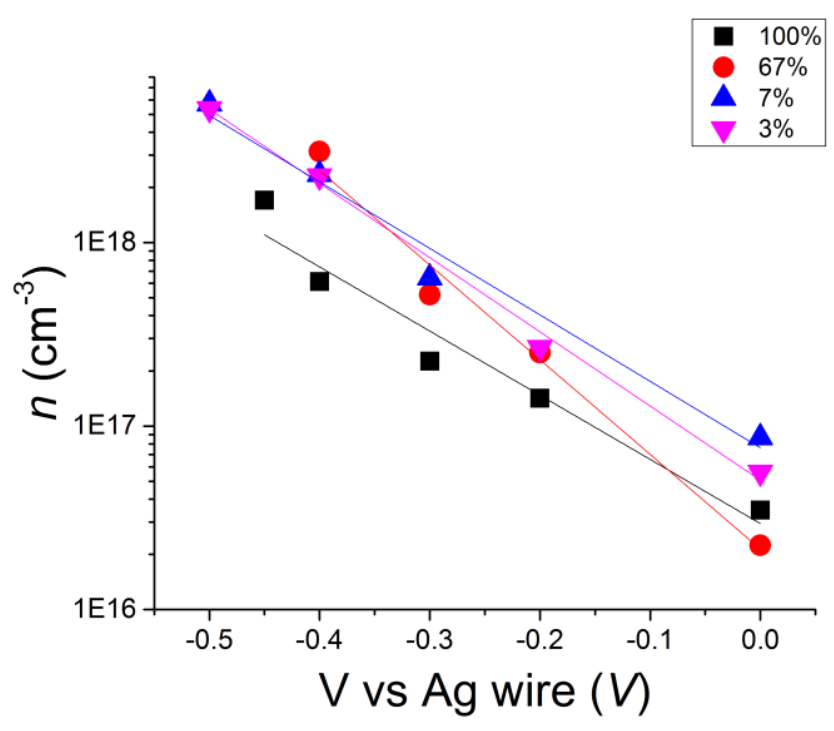

Figure S3. Charge extraction measurements performed for $\mathrm{D} 131$ sensitized $\mathrm{TiO}_{2}$ films under electrochemical bias. The method to extract the electron density is illustrated in figure S2f.

In figure S3 we show the results from the charge extraction measurements. From the slope of electron density versus applied voltage we infer the trap density parameter $\alpha_{T}$ which we use in the simulated of electron hole recombination. For this set of measurements, values of between 0.21 (100\% dye surface coverage) and 0.31 (67\% dye surface coverage) are extracted. $\alpha_{T}=0.2$ is used in the simulations shown in figure $1 \mathrm{~d}$ in the main text and in this document.

\section{Method}

Sample preparation: microscope glass slides or conductive FTO glass slides (TEC-15, purchased from Pilkington) were used as substrates for the samples. The slides were cleaned with soap, rinsed with deionized water, acetone and isopropanol. They finally underwent a heating step at $450^{\circ} \mathrm{C}$ for 30 minutes. $\mathrm{TiO}_{2}$ compact layer spray pyrolysis was performed FTO substrates. The deposition was carried out from a solution of $0.6 \mathrm{ml}$ of titanium isopropoxide (from Sigma Aldrich) in $0.4 \mathrm{ml}$ acetylacetone (Sigma Aldrich) and $9 \mathrm{ml}$ of absolute ethanol. The solution was sprayed on the substrates, kept at $450^{\circ} \mathrm{C}$, using a spray gun fed with compressed air. 4 phases of spraying separated by about 10 seconds were performed. $4 \mathrm{~g}$ of solutions were used when spraying over a surface of about 20 by $30 \mathrm{~cm}^{2}$. The substrates were then left sintering at the same temperature for 30 minutes. This resulted in the formation of a 50 to $100 \mathrm{~nm}$ thick $\mathrm{TiO}_{2}$ compact layer. $\mathrm{TiO}_{2}$ paste was deposited on the glass via doctor blading. The films were sintered at $450^{\circ} \mathrm{C}$ for 30 minutes (10 minutes for the heating ramp) resulting in film thicknesses of about $7 \mu \mathrm{m}$. Samples were stored at $120^{\circ} \mathrm{C}$ and used 
within one day after sintering. The dyeing was performed by immersing the samples in dye solution from the oven. The dyes D131 (purchased from Mitsubishi paper mills limited) and MP13 (see details on synthesis and properties in Section 7 of this document) were used. Full coverage films were dyed using $0.1 \mathrm{mM}$ D131 solution in 1:1 acetonitrile:tert-butyl alcohol or $0.1 \mathrm{mM}$ MP13 dissolved in dichloromethane and by dyeing overnight. Control on the D131 loading was attained by dyeing the film for 145 minutes or by introducing cheno-deoxycholic acid (DCA) in the D131 solution at 1:200 D131:DCA ratio and dyeing for 1 to 2 hours. The chemical structures of the dyes D131, MP13 and the coadsorbent DCA are illustrated in figure 5 in the main text. All samples were then rinsed in neat solvent (the same as the dyeing). Dye desorption was also investigated following the recipe shown in reference ${ }^{4}$. D131 sensitized samples were left in solutions of between 0.05 and $0.2 \mathrm{mM}$ tetra methyl ammonium hydroxide in acetonitrile for 30 minutes and subsequently rinsed in acetonitrile for 15 minutes. Dye desorption was also used to evaluate the dye surface coverage. The samples were left in a solution of tetra methyl ammonium hydroxide in acetonitrile until all dyes desorbed from the $\mathrm{TiO}_{2}$ surface. The surface coverage of each sample was evaluated by measuring the concentration of dyes in the film from the absorption of the solution, the area and thickness of the film and compare it to the case of a fully dyed D131 sample (dyed overnight).

Spectroscopy: a Nd:YAG pumped OPO (Opotek Opolette 355) was used as pump at a repetition rate of $10 \mathrm{~Hz}$ for all experiments. Pump wavelength and fluence were set at $450 \mathrm{~nm}$ and below $5 \mu \mathrm{J} \mathrm{cm}^{-2}$ for D131 samples while $430 \mathrm{~nm}$ and fluence between 2 and $3 \mu \mathrm{J} \mathrm{cm}{ }^{-2}$ were used for MP13 samples. A 100W quartz halogen lamp (Bentham IL1) driven by constant current power supply (Bentham 605) was used as probe light source in the system. Neutral density and long pass filters were used to attenuate the intensity of the probe and to limit its spectral range to wavelengths above the absorption window of the dye. After the sample, the probe beam was passed through a monochromator where the probe wavelength was selected before reaching a visible or an infrared detector. The electrical signal from the detector was amplified via a Costronics preamplifier and amplifier and recorded with an oscilloscope (Tektronix TDS 1012). The amplifier also measured the background level which was used to calculate the variation in optical density upon laser excitation, $\Delta O D$, using the formula $\Delta O D(t)=-\log _{10}\left(1+V(t) / V_{\mathrm{BG}}\right) \approx-V(t) /\left(V_{\mathrm{BG}} \times 2.3\right)$ where $V(t)$ is the transient signal and $V_{\mathrm{BG}}$ the background level. $V(t)$ was evaluated from the measured signal after subtracting a baseline signal measured while blocking the probe beam to correct for electrical interference induced by the pulsed laser and eventual photoluminescence of the sample.

For transient absorption anisotropy measurements, the same setup to the one described above was used. The pump beam was passed through a Glan-Thompson polarizer (GTH10M-A from Thorlabs) before hitting the sample. The polarization of the pump was kept vertical with respect to the plane of the optical table. A second polarizer (LPVIS050 from Thorlabs) was mounted before the monochromator to probe the vertical or horizontal polarization (parallel or perpendicular to the pump beam's polarization) of the transient optical signal transmitted through the sample. $\triangle O D$ was calculated as above for all conditions.

Monte Carlo simulations of anisotropy decay: simulations for D131 dyes on cubic particles were carried out using a modified version of the Monte Carlo code described in reference ${ }^{5}$. The rate of hole transfer between neighbouring dyes was computed using non-adiabatic Marcus theory of charge transfer: 
$\Gamma_{i, j}=\frac{2 \pi}{\hbar} \frac{|J|^{2}}{\sqrt{4 \pi \lambda k T}} e^{-\frac{\left(\lambda+E j-E_{i}\right)^{2}}{4 \lambda k T}}$ Eq. S1

Where $\hbar$ is the reduced Plank's constant, $\lambda$ is the reorganization energy of charge transfer, $J$ is the electronic coupling, $k$ is Boltzmann constant, $T$ is temperature and $E_{i}$ and $E_{j}$ are the site energies of the initial and final states. For simulations of hole diffusion in D131 monolayers the value of the reorganization energy $(\lambda=1.062 \mathrm{eV})$ was calculated using the method presented in reference ${ }^{6}$, while the electronic coupling was estimated from the experimentally determined effective electronic coupling ${ }^{7}\left(J_{\text {eff }}=51 \mathrm{meV}\right)$ and 'distributing' this value over the 8 nearest neighbors considered in this model $\left(J=J_{\text {eff }} / 8^{1 / 2} \sim 18 \mathrm{meV}\right)$.

\section{Analysis of dispersion in transient absorption anisotropy decays}

A mono-exponential transient absorption anisotropy decay is expected for samples where the probed excited states diffuse on the surface of a sphere and the transition dipole moments are perpendicular to the sphere's surface. ${ }^{8}$ Hole diffusion in a dye monolayer sensitizing the surface of a mesoporous $\mathrm{TiO}_{2}$ film results in a transient anisotropy decay which deviates from the monoexponential behaviour and occurs over several orders of magnitude in timescale. Below we describe some of the factors that may underlie this observation.

Some of these factors have no direct connection to the hole transport properties of the dye layer, however they have an influence on the shape of the anisotropy decay. For example:

- Shape and distribution in size of the nanoparticles.

- Orientation of the dyes' transition dipole moments with respect to the surface. This can vary from site to site due to difference in dye configuration.

The implication is that the transient anisotropy measurement is not a direct probe of hole transport. Dye monolayers showing nominally identical hole diffusion properties may show very different anisotropy decay dynamics due to the scaffold geometry and the distribution of transition dipole moment orientations (see below).

On the other hand, other factors influence the shape of the anisotropy decay because they directly change the hole transport properties of the dye monolayer. These are mainly configurational and energetic disorder which contribute to broaden the distribution of charge transfer rates between different dyes, resulting in dispersive hole transport.

In the main text, we discuss the effect of dye surface coverage and energetic disorder on the stretching of the anisotropy decay. Below we show simulations describing these and the other factors listed above. The sources of stretching are considered separately in order to clarify their contribution. The base model for all the calculations is the cubic particle sensitized with dyes arranged in a square lattice as described in the method section. The dyes' transition dipole moments are oriented perpendicular to the particle surface unless stated otherwise. 


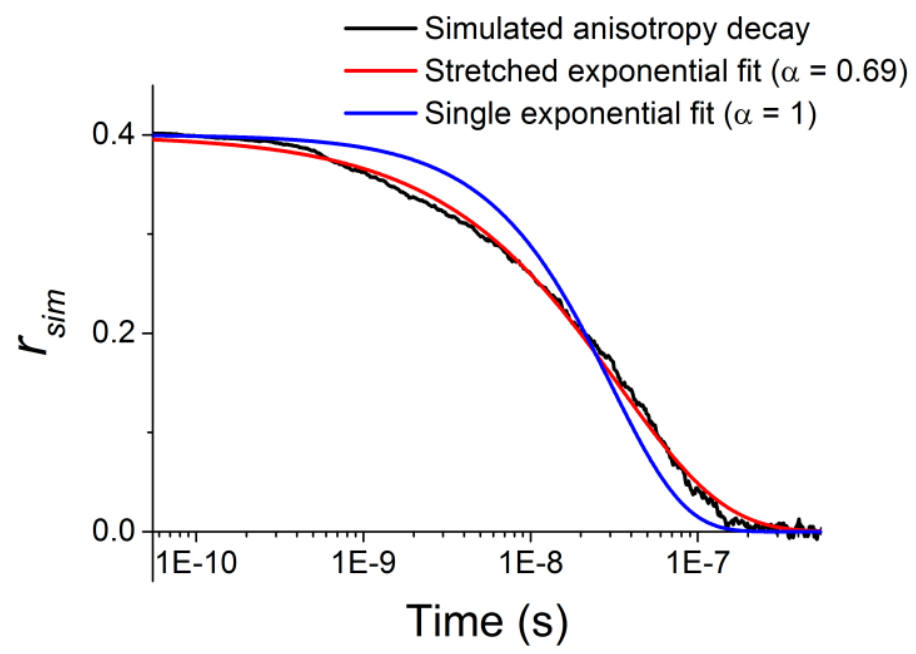

Figure S4. Fit of single exponential and stretched exponential to a simulated anisotropy decay.

Figure S4 shows that the expected anisotropy decay for hole diffusing on a cubic particle does not follow an exponential profile, instead a degree of stretching is introduced by the non-spherical shape of the system. Analogous argument is valid for other non-spherical shapes expected for the $\mathrm{TiO}_{2}$ particles used in our experiments. 


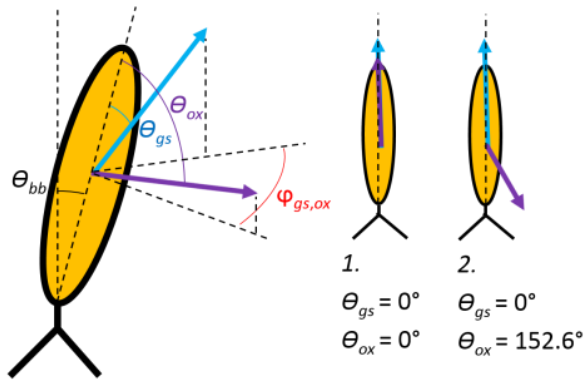

a
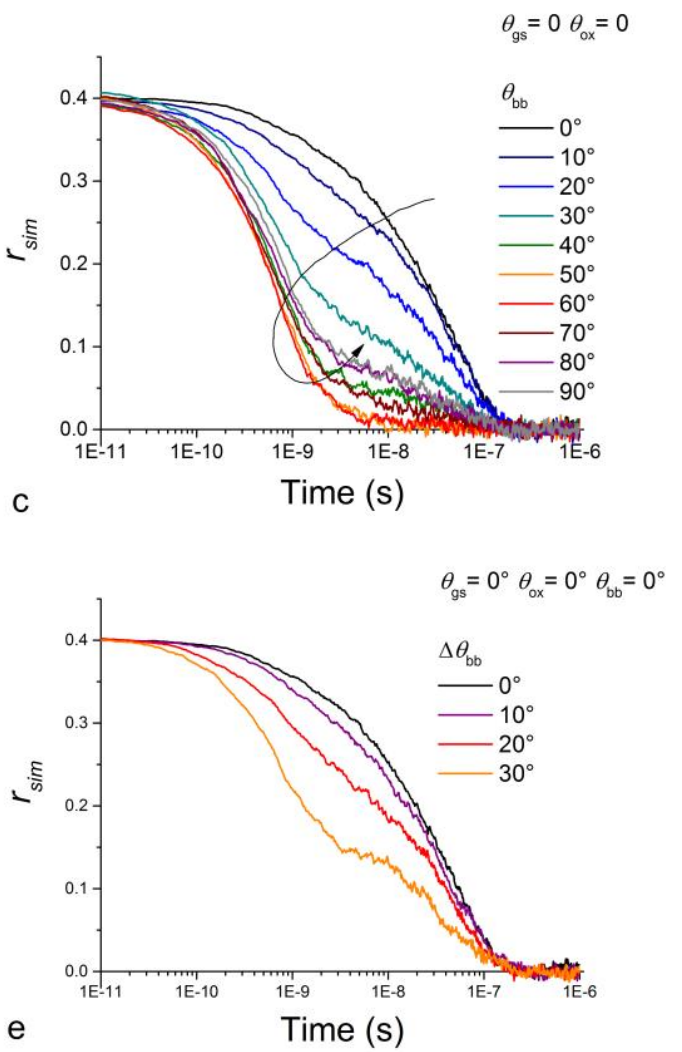

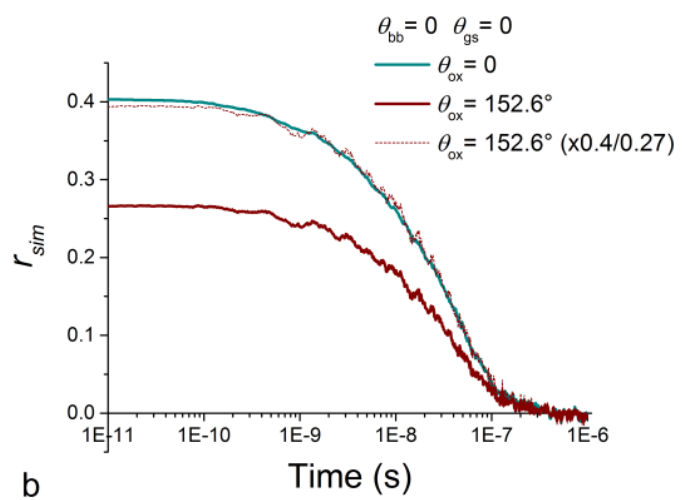

$\theta_{g s}=0 \theta_{o x}=152.6^{\circ}$

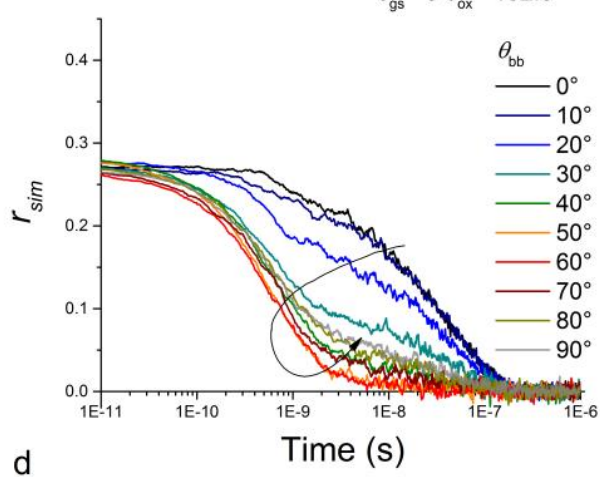

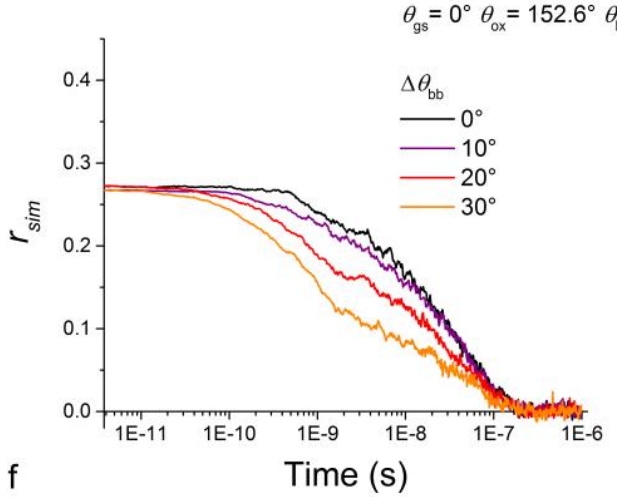

Figure S5. Analysis of the effect of molecular orientation and transition dipole moments orientation on the simulated transient absorption anisotropy decay. (a) Schematics showing our convention for the relevant angles when describing dye configuration. Case 1 and 2 are the ones we focus on to discuss our experimental results. (b) Transient anisotropy simulation for systems with different oxidized dye transition dipole moment orientation. (c) and (d) show the effect of dye backbone orientation on the transient anisotropy dynamics for case 1 and 2. (e) and (f) illustrate the influence of a Gaussian distribution of backbone orientations on the simulated transient anisotropy decay for case 1 and 2 .

In figure S5 we show simulations of transient absorption anisotropy where the dye structure orientation on the surface of the particle and the orientation of the transition dipole moments of the dyes are varied. Figure S5a showed our simplified model for a dye anchored to a surface. A main structural axis (backbone) of the molecule can be defined. We indicate the angle between this axis and the normal to the surface as $\Theta_{b b}$. The angle between the unoxidized dye transition dipole 
moment and dye backbone $\left(\theta_{g s}\right)$ and the angle between the oxidized dye transition dipole moment and the backbone $\left(\theta_{o x}\right)$ are also defined. Finally, the difference in azimuth between the two vectors with respect to the dye backbone is shown as $\varphi_{g s, o x}$. In the simulations shown in figure 5 , dyes are arranged on the surface of the cube with a certain $\theta_{b b}$. Each dye is also rotated by a random angle about the normal to the surface. The orientation of the dipole moments is then defined according to $\theta_{g s}, \theta_{o x}$ and $\varphi_{g s, o x}$ with respect to the backbone axis and finally a rotation about the backbone axis by a random angle is applied. In figure S5 we considered some simple combinations of the available parameters in order to show relevant trends to our study and to the interpretation of our experimental data. In particular, we define the backbone of the dye to be aligned with the transition dipole moment of the unoxidized dye in all cases $\left(\theta_{g s}=0^{\circ}\right)$. This assumption is based on the results displayed in figure S1a. Furthermore we consider the case of (1) $\theta_{o x}=0^{\circ}$, similar to simulations displayed in figure $2 \mathrm{~d}$ and $2 \mathrm{f}$, and the case of (2) $\theta_{o x}=152.6^{\circ}$. While case 1 is chosen for simplicity, case 2 is based on our TD-DFT calculations of the D131 dye. Figure $5 b$ shows that case 2 results in a lower value of the maximum anisotropy than case 1 , as discussed in the main text and in Section 1 of this document. It also shows that the shape of the decay is not different for case 1 and 2 . In figure $5 \mathrm{c}$ and $5 \mathrm{~d}$ we investigate the effect of considering a constant value of $\theta_{g s}$ between $0^{\circ}$ and $90^{\circ}$ for case 1 and 2 respectively. We observe a faster decay in anisotropy when the unoxidized transition dipole moment is not normal to the surface. The decay is fastest for the cases of $\theta_{g s}$ equal to $40^{\circ}$ and $50^{\circ}$. Finally we investigate the influence of a Gaussian distribution in backbone orientation $\theta_{b b}$ centered in $\theta_{b b}=0^{\circ}$ with standard deviation $\Delta \theta_{b b}$ and limited to the range $0^{\circ} \leq \theta_{b b} \leq 90^{\circ}$. An increasingly fast decay in anisotropy is observed for increasing values of $\Delta \theta_{b b}$ both for case 1 (figure S5e) and for case 2 (figure S5f). We stress that all simulations presented in figure S5 correspond to identical hole diffusion performance. We conclude that the interpretation of experimental transient absorption anisotropy decays for dye sensitized systems in terms of hole transfer rate is subject to multiple levels of uncertainty, mainly related to the distribution in surface geometry and monolayer configuration.

Below we consider factors that directly influence the transport properties in the dye monolayer such as the parameters that define the hole transfer rate between dyes.

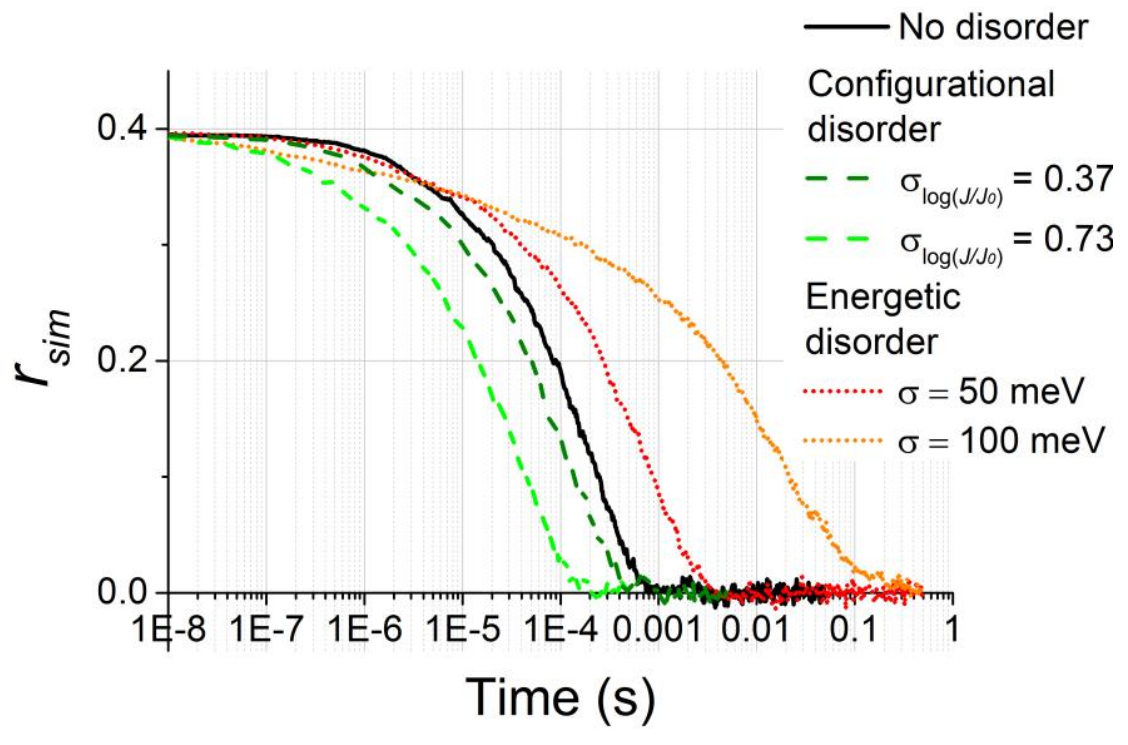


Figure S6. Effect of energetic disorder and disorder in the electronic coupling distribution between dyes on the profile of simulated transient anisotropy. A Gaussian distribution of electronic couplings and site energies with different standard deviations were used.

Figure S6 displays simulations where changes in hole transport due to different sources of disorder are reflected in different simulated anisotropy decay. All simulations consider case 1 (see figure S5a), i.e. all dyes' transition dipole moments are oriented perpendicularly to the cube surface. In this way we can isolate the effect of distributions in site energies and electronic couplings from other effects of configurational disorder discussed above. The black line corresponds to the case of fully covered cubic particle where dyes are coupled to 8 nearest neighbors with electronic coupling $J=2.12810^{-4}$ $\mathrm{eV}$ and reorganization energy $\lambda=1.014 \mathrm{eV}$. The dotted lines correspond to the same system where site energies drawn from a Gaussian distribution with standard deviation $\sigma_{\mathrm{E}}$ have been assigned to each site. This is a first order representation of the energetic disorder present in the dye monolayer. The effect is a pronounced dispersion in the decay. Such dispersion is very sensitive to the value of $\sigma_{\mathrm{E}}$, as also shown in the main text.

The dashed lines show the effect of assigning to each pair of dyes values of electronic couplings drawn from a Gaussian distribution defined on a logarithmic scale with the same mean as the reference simulation and a standard deviation $\sigma_{\log (/ / J)}$. This can be seen as a representation of configurational disorder in the monolayer, due to different inter-dye distances and dye conformations. The effect of introducing a non-zero $\sigma_{\log (/ / / 0)}$ is an acceleration in the decay along with a slight increase in dispersion. The acceleration can be explained by considering that holes have higher chances to find a high coupling 'percolating path' when configurational disorder is included than in a disorder-free monolayer. This decreases the average time that it takes for holes to reach a different facet of the cube from the one where they have been generated. The effect is not observed for the energetic disorder case, as fluctuations in the energy landscape lead to efficient trapping of charges which are then released at longer timescales, resulting in an overall deceleration in the anisotropy decay.
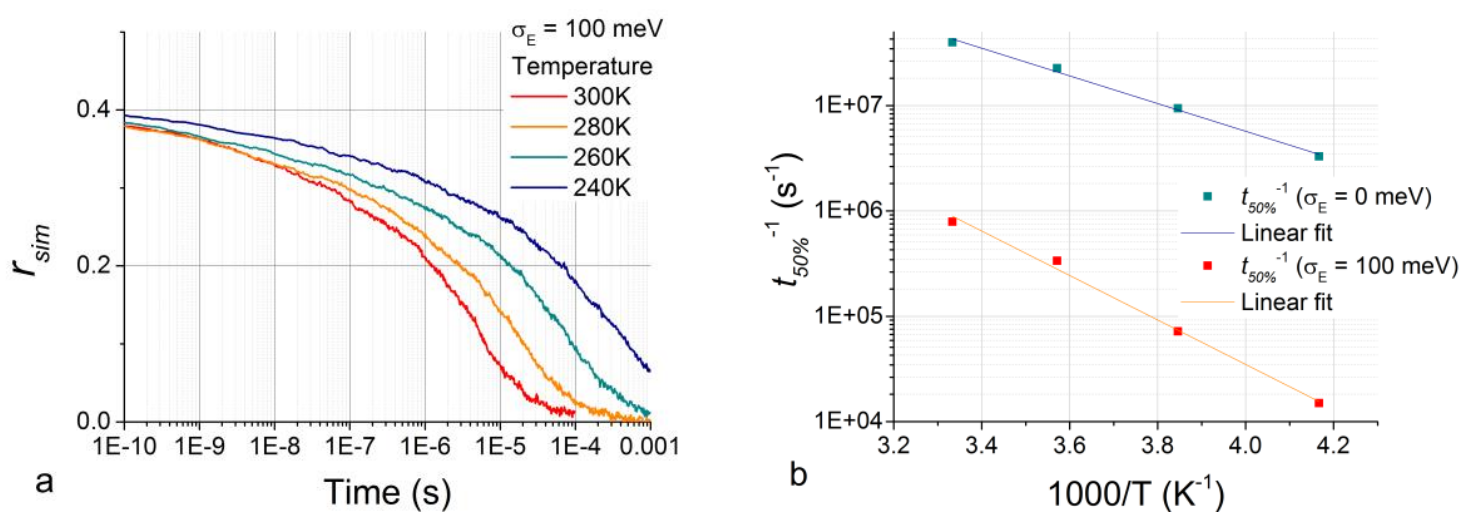

Figure S7. (a) Simulated anisotropy decay for a cubic particle fully covered with D131 dyes where energetic disorder is introduced in the dye monolayer $\left(\sigma_{\mathrm{E}}=100 \mathrm{meV}\right)$. The different traces correspond to different temperatures. (b) Temperature dependence of the $t_{50 \%}{ }^{-1}$ of the transient anisotropy simulated for the case of $\sigma_{\mathrm{E}}=0 \mathrm{meV}$ (no energetic disorder) and $\sigma_{\mathrm{E}}=$ $100 \mathrm{meV}$. 
Figure S7 shows the effect of energetic disorder on the simulated temperature dependent anisotropy decay. $A$ value of $\sigma E=100 \mathrm{meV}$ has been used (as also shown in figure $2 \mathrm{~h}$ in the main text). Figure $\mathrm{S} 7 \mathrm{~b}$ displays $t_{50 \%}{ }^{-1}$ obtained from the anisotropy simulations. Note that $t_{50 \%}$ is not a half life in this case, as anisotropy is not related to the lifetime of the holes, but simply the time at which anisotropy decays by $50 \%$ from the initial value. An apparent reorganization energy of $0.996 \mathrm{eV}$ is found by fitting the data for the disorder-free simulations to non-adiabatic Marcus equation (see equation S1). This value is very close to the input value of $\lambda=1.062 \mathrm{eV}$. For the disordered case, an apparent reorganization energy of $1.72 \mathrm{eV}$ is found. The observed increase is related to the thermal activation needed to escape energetic traps which is expressed in Marcus' formula by the Ej - Ei term. A similar effect could explain the observed difference between measured and calculated reorganization energy that we obtained in our previous investigation (see reference ${ }^{7}$ ).

\section{Simulation of electron hole recombination}
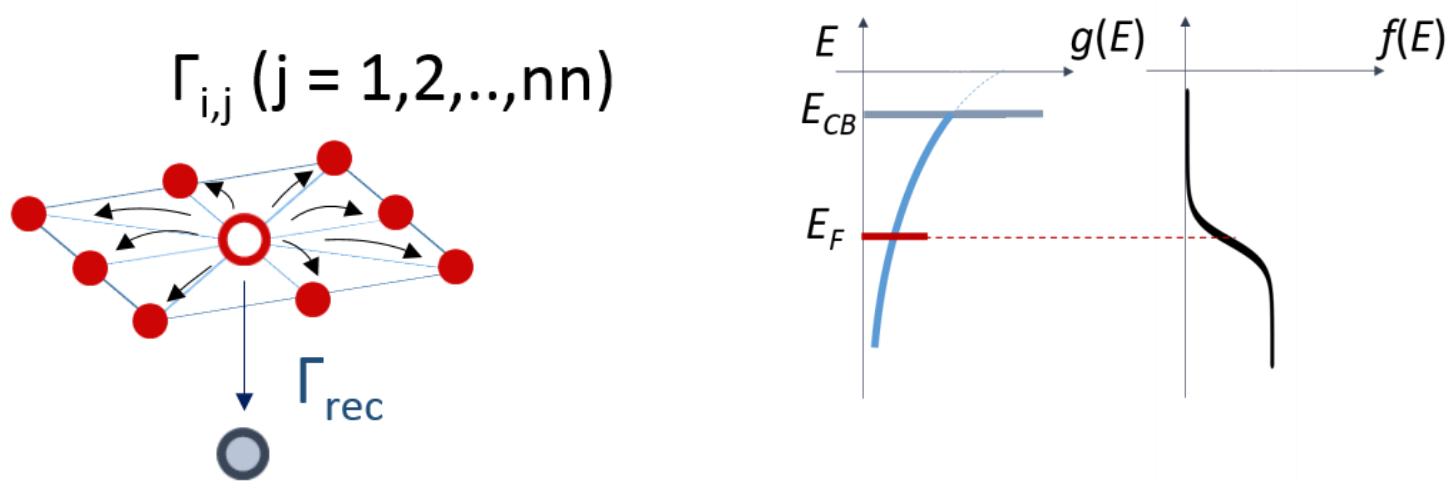

Figure 58. Schematics of the model of hole diffusion and electron hole recombination used in our Monte Carlo simulations. The hole can be transferred to either of the nearest neighbours $(\mathrm{nn})$ of the oxidized dye or it can recombine with an electron occupying the state associated to the oxidized dye site.

Figure $\mathrm{S} 8$ shows a schematics of the model used in this work (figure $1 \mathrm{~d}$ in the main text and figures in this section) to simulate electron-hole recombination. A hole on a dye can hop to either of the neighbouring sites (if occupied by a dye) or can recombine with an electron occupying the electronic state associated to the dye site. Electronic energy for the $\mathrm{TiO}_{2}$ surface states are drawn from an exponential distribution, consistently with the experimentally extracted density of states $g(E) \sim \exp (-$ $\left.\alpha_{T} E / k T\right)$. Here, the assumption is that each dye can interact with only one electronic state in the $\mathrm{TiO}_{2}$. The position of the conduction band edge in the $\mathrm{TiO}_{2}$ is not known. Below we discuss how this parameter influences the simulated results.

The rates involved in the hole random walk and recombination process are calculated as follows:

- The rate of hole transfer to neighbouring dyes $\Gamma_{i, j}$ are calculated following equation $\mathrm{S} 1$ given the initial and final state of the reaction.

- The rate of electron hole recombination is calculates as $\Gamma_{\text {rec }}=k_{e d r} \Gamma_{0} f\left(E_{T, i}\right)$. $\Gamma_{0}$ is a reference value of rate of hole hopping between dyes calculated in the particular case of $E_{\mathrm{f}}=E_{\mathrm{i}} . \mathrm{f}\left(E_{T, i}\right)$ is the Fermi-Dirac occupancy probability for the electronic state with energy $E_{T, i}$ associated to 
the $i$ dye site where the hole is, given the input Fermi level position. $k_{e d r}$ is a free parameter in the model, used to investigate the relative order of magnitude between $\Gamma_{\text {rec }}$ and $\Gamma_{j}$. We note that a more accurate model describing recombination if holes to trapped electrons would consider an energy dependence of the rate. In this model the only dependence from energy in the calculation of the rate is included in the Fermi-Dirac term.

$\Gamma_{\text {rec }}$ and $\Gamma_{i, j}$ contributes to the calculation of the waiting time according to

$$
t_{\text {wait }}=-\frac{\ln (R)}{\Gamma_{r e c}+\sum_{j} \Gamma_{i, j}}, \quad \text { Eq. S2 }
$$

where $R$ is a random number between 0 and 1 . After $t_{\text {wait, }}$, the hole either hops to a neighbouring site or recombines with the electron. The probability of each event (destination) to be picked is proportional to the rate associated to that event divided by the sum of all rates involved in the calculation of $t_{\text {wait. }}$.
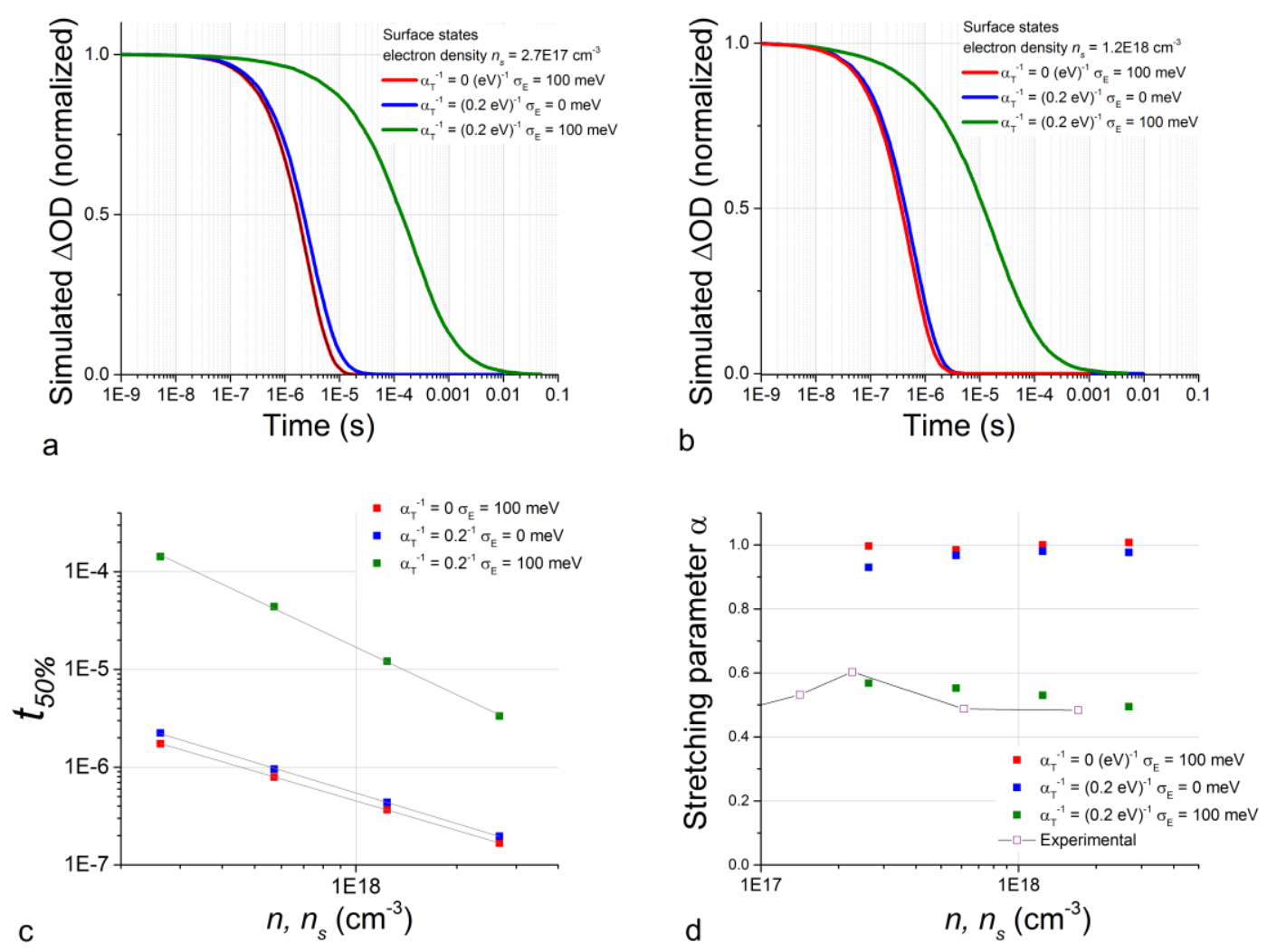

Figure S9. Investigation of the effect of energetic disorder in the dye monolayer and trap density distribution in the $\mathrm{TiO}_{2}$ on the dynamics of electron hole recombination. Figures $\mathrm{a}, \mathrm{b}$ show the influence of energetic disorder between dyes and of trap states in the $\mathrm{TiO}_{2}$ either considered separately or simultaneously on the simulated recombination kinetics. The (c) simulated hole lifetime and (d) stretching parameter obtained by fitting a stretched exponential to the simulated decays are also displayed.

In figure S9 we show the computational analysis of the recombination mechanism between electrons and photogenerated hole when energetic disorder in the dye monolayer and in the $\mathrm{TiO}_{2}$ surface 
states are considered either separately or simultaneously. As also displayed in table S1, when the two conditions are considered in combination, the experimental stretching of the hole population decay and the electron density dependence of the hole $t_{50 \%}$ are approximately matched. When only either energetic disorder in the dye monolayer or in the $\mathrm{TiO}_{2}$ surface is considered, we observe nearly monoexponential decay of the hole population and $t_{50 \%} \sim n^{-1}$.

Table S1. Charge density dependence of lifetime and stretching parameter for the data displayed in figure S9

\begin{tabular}{llll}
\hline $\boldsymbol{\alpha}_{T^{-1}}\left(\mathrm{eV}^{-1}\right)$ & $\sigma_{\mathrm{E}}(\mathrm{meV})$ & Slope $\log \left(t_{50 \%}\right)$ vs $\log (\boldsymbol{n})$ & Stretching parameter $\boldsymbol{\alpha}$ (from fit) \\
\hline 0 & 100 & $0.99^{-1}$ & $0.98-1.01$ \\
$0.2^{-1}$ & 0 & $0.96^{-1}$ & $0.93-0.98$ \\
$0.2^{-1}$ & 100 & $0.61^{-1}$ & $0.45-0.55$ \\
Experimental & & $0.53^{-1}$ & $0.40-0.60$ \\
\hline
\end{tabular}
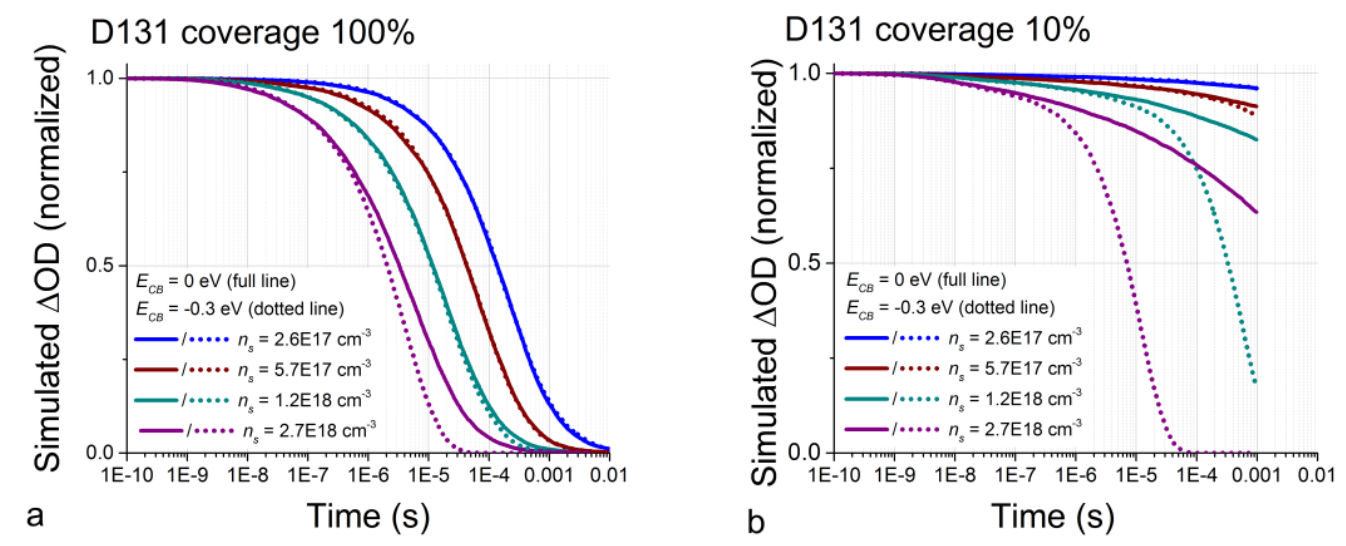

Figure S10. Simulated electron-hole recombination for $\mathrm{D} 131$ sensitized $\mathrm{TiO}_{2}$ under (a) $100 \%$ dye surface coverage and (b) $10 \%$ dye surface coverage, for different values of electron density in the particle and for two values of the conduction band edge.

In figure S10 we show results from our electron-hole recombination model for the cases of complete and $10 \%$ dye surface coverage. The data indicate a slowdown in recombination when low surface coverage is adopted. This is because holes are not able to diffuse to surface states with higher electron occupancy probability. We also show that the position of the conduction band edge, as we define it in this model (see description above), has an important influence on the lifetime for low dye coverage conditions, while it has only minor effect for the complete coverage case. We note that the dynamics presented for the low coverage simulations poorly reproduce experimental observations. 


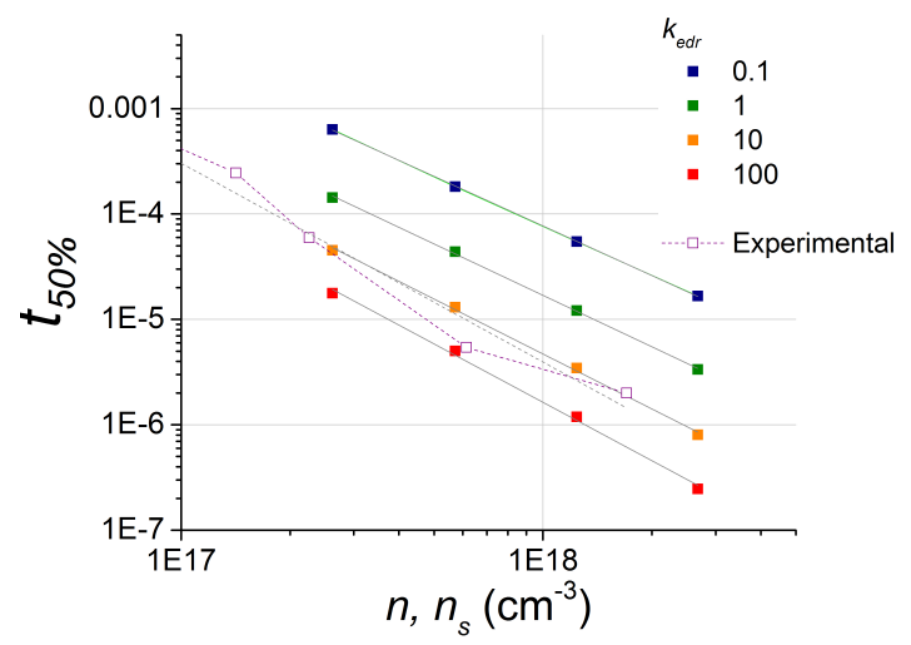

Figure S11. Simulated hole lifetime corresponding to different values of the free parameter $k_{\text {edr }}$. This parameter expresses the relative magnitude of electron transfer from $\mathrm{TiO}_{2}$ to an oxidized dye and hole hopping between dyes. The experimental lifetime is also displayed.

The other free parameter in this electron-hole recombination model is kedr, which effectively represents the relative rate of charge transfer between neighbouring dyes and oxidized dyes to occupied $\mathrm{TiO}_{2}$ surface states. In figure $\mathrm{S} 11$ and table $\mathrm{S} 2$ we show that by varying such parameter, the electron density dependence and the stretching parameter obtained by fitting a stretched exponential to the simulated hole population decays is approximately maintained.

Table S2. Charge density dependence of lifetime and stretching parameter for the data displayed in figure S11

\begin{tabular}{lll}
\hline Conditions & Slope $\log \left(t_{50 \%}\right)$ vs $\log (\boldsymbol{n})$ & Stretching parameter $\boldsymbol{\alpha}$ (from fit) \\
\hline$k_{\text {edr }}=0.1$ & $0.64^{-1}$ & $0.54-0.59$ \\
$k_{e d r}=1$ & $0.61^{-1}$ & $0.49-0.57$ \\
$k_{e d r}=10$ & $0.58^{-1}$ & $0.45-0.55$ \\
$k_{\text {edr }}=100$ & $0.54^{-1}$ & $0.43-0.53$ \\
Experimental & $0.53^{-1}$ & $0.40-0.60$ \\
\hline
\end{tabular}

\section{Dye degradation}

Photo-oxidation of the sensitizers in the experiments presented in this work is followed by recombination with electrons injected in $\mathrm{TiO}_{2}$. However, dye degradation and dye desorption from the surface represent alternative 'relaxation routes'. Variation in the optical absorption of the sample 
before and after the measurements was observed for some samples, suggesting that either or both processes occurred to some extent during the measurement.
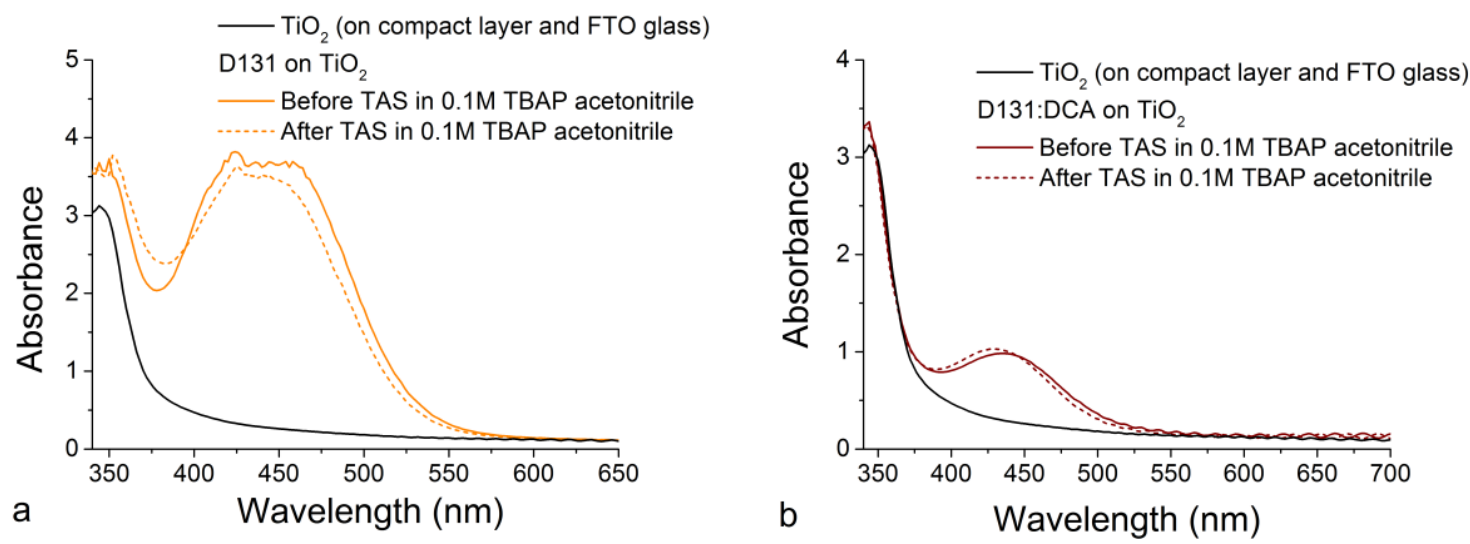

Figure S12. Degradation of (a) short dyed D131 (67\% coverage) and (b) D131:DCA on $\mathrm{TiO}_{2}$ measured in 0.1M TBAP acetontrile.

Figure $\mathrm{S} 12$ shows UVvis spectra for $\mathrm{D} 131$ and D131:DCA sensitized $\mathrm{TiO}_{2}$ films. Variation in magnitude and spectral shifts are observed. In particular for figure S12a, by considering the absorbance drop and an estimate of the total number of photons incident on the sample during the measurements, we quantify an upper limit in the yield of degradation in the order of 0.1 .

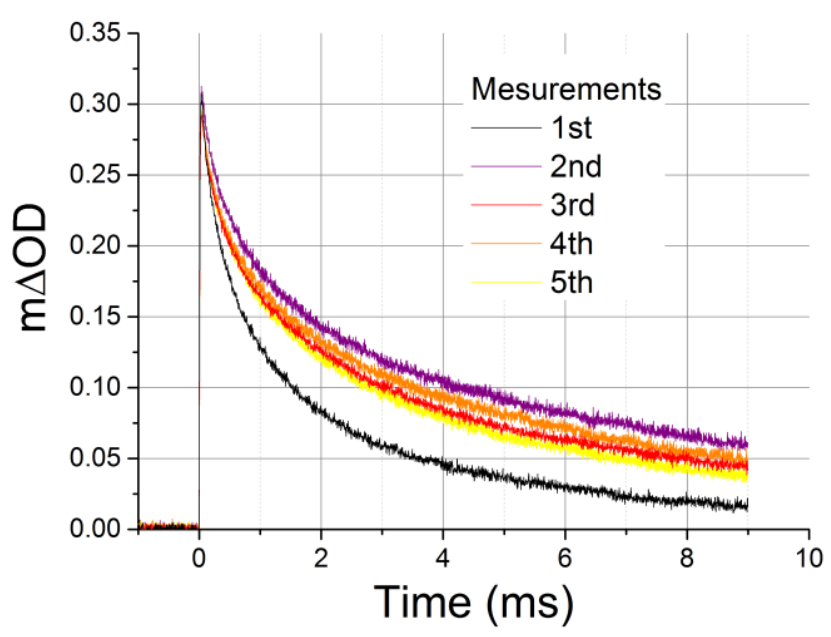

Figure S13. TAS decays measured for $\mathrm{D} 131$ sensitized $\mathrm{TiO}_{2}$ films in $0.1 \mathrm{M}$ TBAP in acetonitrile. The 5 measurements presented were performed over a period of about 4 hours.

The transient absorption decay was also found to undergo changes during the measurements. This suggests that prolonged photo-excitation of the samples influences the kinetics of recombination, possibly due to variations in the surface properties (see an example in figure S13). 

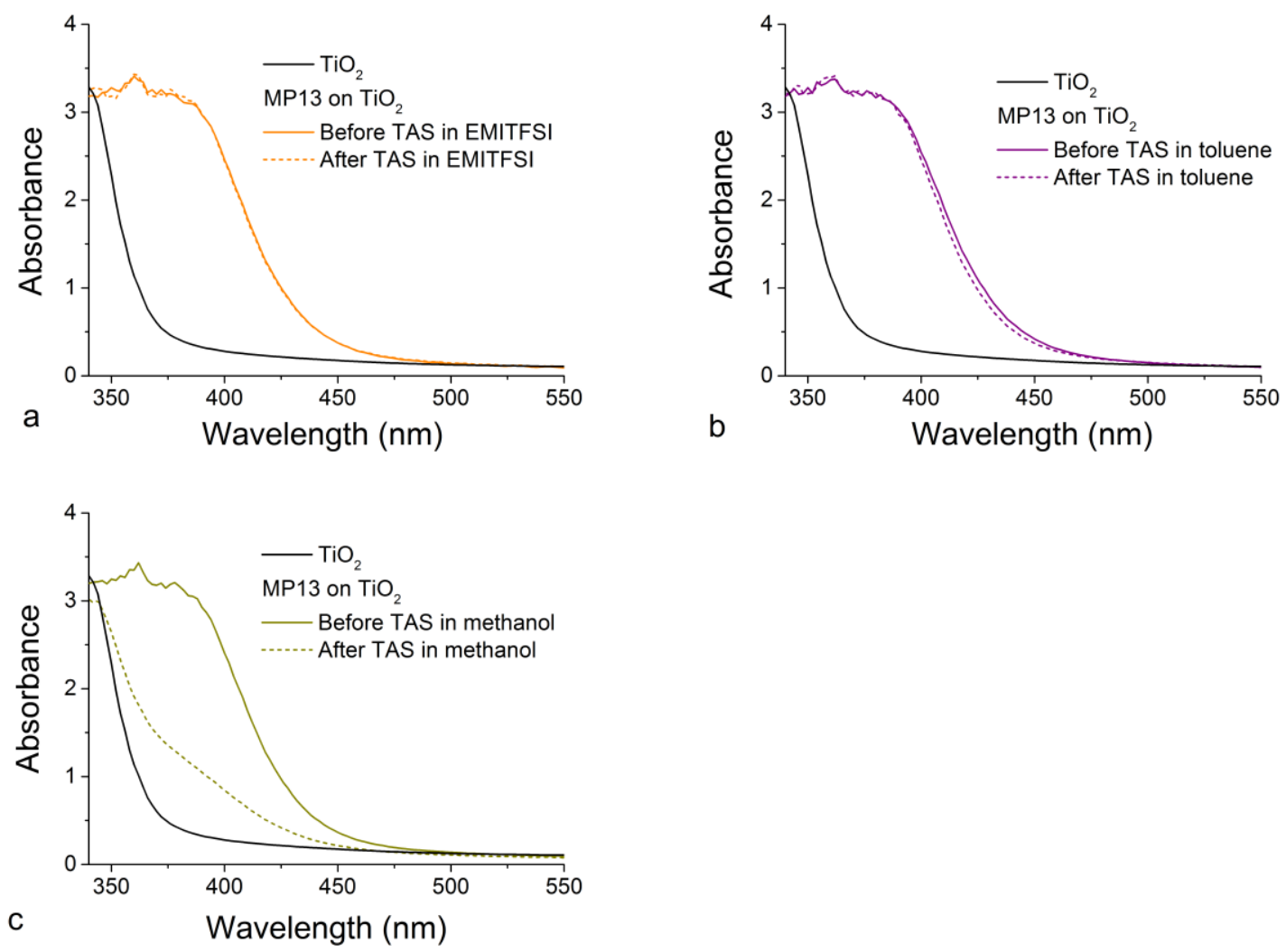

Figure S14. UV-vis absorbance spectra of $\mathrm{MP13}$ on $\mathrm{TiO}_{2}$ before and after the TAS measurements performed in different solvents. Examples of (a) negligible degradation (EMITFSI), (b) limited degradation (toluene) and (c) severe degradation (methanol).

Figure S14 shows the different degrees of degradation observed for MP13 sensitized films measured in different solvents. For the case of methanol and ethylene glycol, severe decrease in absorbance was observed. Also, TAS decays for these samples followed different dynamics from the ones reported in figure $4 a$ in the main text, suggesting that other processes may occur upon photooxidation of the dye, such as oxidation of solvent molecules. ${ }^{9}$

\section{MP13 Synthesis and Properties}

Materials. All reagents were purchased from either Sigma-Aldrich or Alfa-Aesar and they were used as received without further purification unless otherwise stated. Terminal acetylene triphenylamine derivative 1 was synthesized following the procedure described previously by our group. ${ }^{10}$
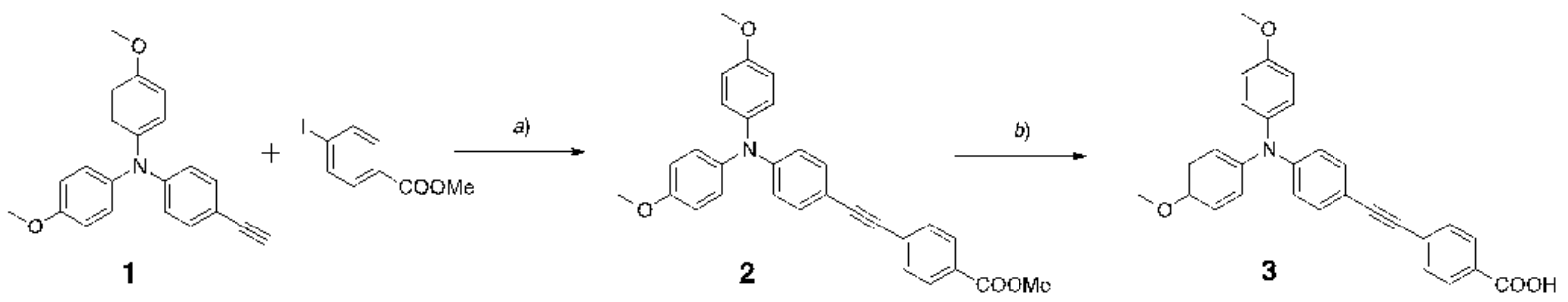

Figure S15. Synthesis of MP13. Reaction conditions $a)\left(\mathrm{PPh}_{3}\right)_{2} \mathrm{PdCl}_{2}, \mathrm{Cul}, \mathrm{PPh}_{3}$, piperidine, toluene; $\left.b\right)$ $\mathrm{LiOH}, \mathrm{THF}, \mathrm{MeOH}, \mathrm{H}_{2} \mathrm{O}$. 
Synthesis of methyl 4-((4-(bis(4-methoxyphenyl)amino)phenyl)ethynyl)benzoate (2). 1 (186 mg, $0.567 \mathrm{mmol}$ ), methyl 4-iodobenzoate $(147 \mathrm{mg}, 0.567 \mathrm{mmol})$, bis(triphenylphosphine)palladium dichloride (10 $\mathrm{mg}, 0.014 \mathrm{mmol}$ ) triphenylphosphine $(7 \mathrm{mg}, 0.028 \mathrm{mmol}$ ) and copper iodide (11 $\mathrm{mg}$, $0.057 \mathrm{mmol}$ ) were placed in a shlenck tube and dried under high vacuum for 30 minutes. Then, a previously degassed solution of dry piperidine $(220 \mu \mathrm{L}, 2.264 \mathrm{mmol})$ in dry toluene $(3 \mathrm{~mL})$ was added via cannula. The mixture was degassed again by the pump/freeze technique and stirred at $75^{\circ} \mathrm{C}$ overnight under $\mathrm{N}_{2}$. Then, a silica plug was run in $\mathrm{CH}_{2} \mathrm{Cl}_{2}$ and the solvent removed. The crude was purified by column chromatography $\left(\mathrm{SiO}_{2}, \mathrm{CH}_{2} \mathrm{Cl}_{2}\right)$ to afford the product as yellow oil, which solidify slowly (177 mg, 67\% yield).

${ }^{1} \mathrm{H}$ NMR $\left(500 \mathrm{MHz}, \mathrm{CDCl}_{3}\right) \delta_{\mathrm{H}}: 7.99(\mathrm{~d}, \mathrm{~J}=8.1,2 \mathrm{H}) ; 7.54(\mathrm{~d}, \mathrm{~J}=8.1,2 \mathrm{H}) ; 7.31(\mathrm{~d}, \mathrm{~J}=8.1,2 \mathrm{H}) ; 7.08(\mathrm{~d}, \mathrm{~J}$ $=8.9,4 \mathrm{H}) ; 6.85(\mathrm{~d}, \mathrm{~J}=8.9,4 \mathrm{H}) ; 6.84(\mathrm{~d}, \mathrm{~J}=8.1,2 \mathrm{H}) ; 3.92(\mathrm{~s}, 3 \mathrm{H}) ; 3.80(\mathrm{~s}, 6 \mathrm{H})$.

${ }^{13} \mathrm{C} \mathrm{NMR}\left(125 \mathrm{MHz}, \mathrm{CDCl}_{3}\right) \delta_{\mathrm{c}}: 166.9 ; 156.6 ; 149.4 ; 140.2 ; 132.8 ; 131.4 ; 129.7 ; 129.0 ; 128.9 ; 127.4$; $119.1 ; 115.0 ; 113.2 ; 93.6 ; 87.7 ; 55.7 ; 52.4$.

MS El (m/z): [M] $]^{+}$calcd for $\mathrm{C}_{30} \mathrm{H}_{25} \mathrm{NO}_{4}: 463.17781$; found: 463.177737 .

Anal. calcd for $\mathrm{C}_{30} \mathrm{H}_{25} \mathrm{NO}_{4}: \mathrm{C}, 77.74 ; \mathrm{H}, 5.44 ; \mathrm{N}, 3.02$; found: $\mathrm{C}, 77.87 ; \mathrm{H}, 5.49 ; \mathrm{N}, 3.17$.

Synthesis of 4-((4-(bis(4-methoxyphenyl)amino)phenyl)ethynyl)benzoic acid (3). 2 (177 mg, 0.382 $\mathrm{mmol}$ ) was placed in a round bottom flask with $4 \mathrm{~mL}$ of THF and $2.3 \mathrm{~mL}$ of $\mathrm{MeOH}$. Then, $1.5 \mathrm{~mL}$ of aqueous $\mathrm{LiOH} 1 \mathrm{M}$ solution was added and the mixture was stirred overnight at r.t. under $\mathrm{N}_{2}$. The crude was extracted with $100 \mathrm{~mL}$ of $\mathrm{CHCl}_{3}$ and washed with citric acid $10 \%$ (100 mL), distilled water $(100 \mathrm{~mL})$ and brine $(100 \mathrm{~mL})$. The organic layer was dried over $\mathrm{Na}_{2} \mathrm{SO}_{4}$, filtered off and solvent removed, to afford the product as yellow solid (167 $\mathrm{mg}, 98 \%$ ).

${ }^{1} \mathrm{H} \mathrm{NMR}\left(500 \mathrm{MHz}, \mathrm{CDCl}_{3}\right) \delta_{\mathrm{H}}: 8.05(\mathrm{~d}, \mathrm{~J}=8.2,2 \mathrm{H}) ; 7.57(\mathrm{~d}, \mathrm{~J}=8.2,2 \mathrm{H}) ; 7.32(\mathrm{~d}, \mathrm{~J}=8.0,2 \mathrm{H}) ; 7.08$ (d, J $=9.0,4 \mathrm{H}) ; 6.86(\mathrm{~d}, \mathrm{~J}=9.0,4 \mathrm{H}) ; 6.85(\mathrm{~d}, \mathrm{~J}=8.8,2 \mathrm{H}) ; 3.81(\mathrm{~s}, 6 \mathrm{H})$.

${ }^{13} \mathrm{C}$ NMR $(125 \mathrm{MHz}, \mathrm{CDCl})$ ) $\delta_{\mathrm{c}}: 169.8 ; 156.7 ; 149.5 ; 140.2 ; 132.8 ; 131.5 ; 130.3 ; 129.8 ; 127.8 ; 127.5$; $119.0 ; 115.1 ; 113.1 ; 94.2 ; 87.7 ; 55.72$.

MS El (m/z): [M] $]^{+}$calcd for $\mathrm{C}_{29} \mathrm{H}_{23} \mathrm{NO}_{4}$ : 449.16216; found: 449.162392 .

\section{Methods.}

Chemical characterization. ${ }^{1} \mathrm{H}$ and ${ }^{13} \mathrm{C}$ NMR spectra were recorded on Bruker Advance 500 spectrometer $\left(500 \mathrm{MHz}\right.$ for ${ }^{1} \mathrm{H}$ and $125 \mathrm{MHz}$ for $\left.{ }^{13} \mathrm{C}\right)$. The deuterated solvents are indicated; chemical shifts, $\delta$, are given in ppm, referenced to the solvent residual signal $\left({ }^{1} \mathrm{H},{ }^{13} \mathrm{C}\right)$. Coupling constants $(J)$ are given in $\mathrm{Hz}$. MS were recorded on ThermoElectron MAT 900 using electron impact (EI) ionization technique. Elemental analyses were carried out by Stephen Boyer at London Metropolitan University using a Carlo Erba CE1108 Elemental Analyzer. 
$\mathrm{E}_{\mathrm{ox}}(1)=+0.34 \mathrm{~V}$ (reversible); $\mathrm{E}_{\mathrm{Ox}}(2)=+0.94 \mathrm{~V}$ (irreversible), against ferrocene/ferrocenium internal standard.

All cyclic voltammetry measurements were carried out in freshly distilled $\mathrm{CH}_{2} \mathrm{Cl}_{2}$ using $0.3 \mathrm{M}$ [TBA] $\left[\mathrm{PF}_{6}\right]$ electrolyte in a three-electrode system, with each solution being purged with $\mathrm{N}_{2}$ prior to measurement. The working electrode was a Pt disk. The reference electrode was $\mathrm{Ag} / \mathrm{AgCl}$ and the counter electrode was a Pt rod. All measurements were made at r.t. using an $\mu$ AUTOLAB Type III potentiostat, driven by the electrochemical software GPES. Cyclic voltammetry (CV) measurements used scan rates of $25,50,100,200$ and $500 \mathrm{mV} / \mathrm{s}$, and square wave voltammetry (SWV) was carried out at a step potential of $4 \mathrm{mV}$, a square wave amplitude of $25 \mathrm{mV}$ and a square wave frequency of $15 \mathrm{~Hz}$, giving a scan rate of $40 \mathrm{mV} / \mathrm{s}$. Ferrocene was used as internal standard in each measurement.

\section{Optical characterization.}

Absorbance: $\lambda_{\max }=379 \mathrm{~nm}, \varepsilon=12500 \mathrm{M}^{-1} \mathrm{~cm}^{-1}$

Emission: $\lambda_{\max }=595 \mathrm{~nm}$ (excitation at $380 \mathrm{~nm}$ )

Solution UV-Visible absorption spectra were recorded using Jasco V-670 UV/Vis/NIR spectrophotometer controlled using the SpectraManager software. Photoluminescence (PL) spectra were recorded with Fluoromax-3 fluorimeter controlled by the ISAMain software. All samples were measured in a $1 \mathrm{~cm}$ cell at r.t. with dichloromethane as a solvent. Concentration of $2 \cdot 10^{-5} \mathrm{M}$ and $5 \cdot 10$

${ }^{6} \mathrm{M}$ were used for UV/Visible and $\mathrm{PL}$, respectively.

\section{Supporting references}

(1) Moia, D.; Cappel, U. B.; Leijtens, T.; Li, X.; Telford, A. M.; Snaith, H. J.; O’Regan, B. C.; Nelson, J.; Barnes, P. R. F. J. Phys. Chem. C 2015, 33 (119), 18975.

(2) Anderson, A. Y.; Barnes, P. R. F.; Durrant, J. R.; O’Regan, B. C. J. Phys. Chem. C 2010, 114, 1953.

(3) Lakowicz, J. R. Principles of Fluorescence Spectroscopy, 3rd ed.; Springer Science + Business Media: New York, 2006.

(4) O’Regan, B.; Li, X.; Ghaddar, T. Energy Environ. Sci. 2012, 5 (5), 7203.

(5) Vaissier, V.; Mosconi, E.; Moia, D.; Pastore, M.; Frost, J. M.; De Angelis, F.; Barnes, P. R. F.; Nelson, J. Chem. Mater. 2014, 26, 4731.

(6) Vaissier, V.; Barnes, P.; Kirkpatrick, J.; Nelson, J. Phys. Chem. Chem. Phys. 2013, 15 (13), 4804.

(7) Moia, D.; Vaissier, V.; López-Duarte, I.; Torres, T.; Nazeeruddin, M. K.; O’Regan, B. C.; Nelson, J.; Barnes, P. R. F. Chem. Sci. 2014, 5 (1), 281.

(8) Ardo, S.; Meyer, G. J. J. Am. Chem. Soc. 2011, 133 (39), 15384.

(9) Liao, H.; Qiu, Z.; Wan, Q.; Wang, Z.; Liu, Y.; Yang, N. Appl. Mater. Interfaces 2014, 6, 18055.

(10) Planells, M.; Abate, A.; Hollman, D. J.; Stranks, S. D.; Bharti, V.; Gaur, J.; Mohanty, D.; Chand, S.; Snaith, H. J.; Robertson, N. J. Mater. Chem. A 2013, 1 (23), 6949. 
